\title{
Tonic inhibition of the chloride/proton antiporter ClC-7 by PI(3,5)P2 is crucial for lysosomal pH maintenance
}

Xavier Leray ${ }^{1 *}$, Jacob Hilton ${ }^{1 *}$, Kamsi Nwangwu ${ }^{1}$, Alissa Becerril ${ }^{1}$, Vedrana Mikusevic ${ }^{1}$, Gabriel Fitzgerald ${ }^{1}$, Anowarul Amin $^{1}$, Mary R. Weston ${ }^{1}$, Joseph A. Mindell ${ }^{1}$

${ }^{1}$ Membrane Transport Biophysics Section, National Institute of Neurological Disorders and Stroke, NIH, Bethesda, MD 20892, USA.

*co-first authors

Correspondence to Joseph A. Mindell: mindellj@ninds.nih.gov

Abbreviations used: OG, Oregon Green-488; PI(3,5)P2, phosphatidylinositol-3,5-biphosphate; PI3P, phosphatidylinositol-3-phosphate; BafA1, BafA1; SEM, standard error of the mean. 


\section{ABSTRACT}

The acidic luminal $\mathrm{pH}$ of lysosomes, maintained within a narrow range, is essential for proper degrative function of the organelle and is generated by the action of a V-type $\mathrm{H}^{+}$ATPase, but other pathways for ion movement are required to dissipate the voltage generated by this process. $\mathrm{ClC}-7, \mathrm{a} \mathrm{Cl}^{-}$ $/ \mathrm{H}^{+}$antiporter responsible for lysosomal $\mathrm{Cl}^{-}$permeability, is a candidate to contribute to the acidification process as part of this "counterion pathway". The signaling lipid PI(3,5)P2 modulates lysosomal dynamics, including by regulating lysosomal ion channels, raising the possibility that it could contribute to lysosomal pH regulation. Here we demonstrate that depleting $\mathrm{PI}(3,5) \mathrm{P} 2$ by inhibiting the PIKfyve kinase causes lysosomal hyperacidification, primarily via an effect on CIC-7. We further show that $\mathrm{PI}(3,5) \mathrm{P} 2$ directly inhibits $\mathrm{ClC}-7$ transport and that this inhibition is eliminated in a disease-causing gainof-function ClC-7 mutation. Together these observations suggest an intimate role for $\mathrm{ClC}-7$ in lysosomal $\mathrm{pH}$ regulation.

\section{KEYWORDS}

Lysosome, pH, acidity, Chloride, phosphoinositide, PI(3,5)P2, apilimod, PIKfyve

\section{INTRODUCTION}

Lysosomes are acidic organelles that participate in cellular defense and homeostasis by clearing and recycling intra- and extra-cellular components and informing the cell on its stress and metabolic status (Levine et al., 2011; Saftig and Klumperman, 2009; Settembre et al., 2013). Their acidity provides an optimal environment for luminal enzyme activity and is a source of electrochemical energy for Iysosomal transporters (Müller et al., 2012; Sagné and Gasnier, 2008). Two main systems act in tandem to maintain a set point value ranging between 4.5 and $5.0 \mathrm{pH}$ units: a V-ATPase that pumps and concentrates protons inside the lysosomal lumen using ATP hydrolysis, and a counterion pathway that dissipates the membrane electrical potential generated by V-ATPase activity (Ishida et al., 2013; Mindell, 2012). We currently don't know if the counterion pathway is a single system or a set of channels and transporters with different ion selectivities. One candidate is the lysosomal transporter CIC-7 of the CLC family (Kornak et al., 2001): it is expressed widely, located predominantly on late-endosome/lysosomal membranes, exports $1 \mathrm{H}^{+}$to import $2 \mathrm{Cl}^{-}$per transport cycle and is the major lysosomal chloride conductance (Graves et al., 2008; Lange et al., 2006; Leisle et al., 2011). Although ClC-7's role as a counterion pathway is still debated (Graves et al., 2008; Ishida et al., 2013; Jentsch and Pusch, 2018; Kasper et al., 2005; Kornak et al., 2001; Mindell, 2012; Steinberg et al., 2010; Weinert et al., 2014), new 
work on patients with a novel ClC-7 disease-causing mutation strongly supports this hypothesis, with a gain of function of the transporter resulting in lysosomal hyperacidification (Nicoli et al., 2019).

Recent work established a connection between ClC-7 and the phospholipid kinase PIKfyve (Gayle et al., 2017). PIKfyve synthesizes phosphatidylinositol-3,5-biphosphate (PI(3,5)P2) from PI3P at the lysosomal membrane and participates in the maturation and trafficking of endosomes and lysosomes (Hasegawa et al., 2017; Jin et al., 2016). While it is well established that pharmacological inhibition or gene disruption of PIKfyve depletes PI(3,5)P2, alters endolysosomal trafficking, and leads to generation of large cytoplasmic vacuoles (Bissig et al., 2017; Chow et al., 2007; Choy et al., 2018; Compton et al., 2016; Ikonomov et al., 2001; Sharma et al., 2019; Yamamoto et al., 1995; Zolov et al., 2012), relatively few studies have examined the effects of PIKfyve inhibition on lysosomal pH. In these studies, PIKfyve inhibition had no effect in some cell types but did affect the $\mathrm{pH}$ in others, depending on the inhibitor used and the measurement method (Ho et al., 2015; Sharma et al., 2019). In this context it was surprising that CICN7 and OSTM1 (CIC-7's $\beta$-subunit) were among only four hits in a global CRISPR screen targeting 19,050 protein coding genes to confer apilimod resistance, and the only two genes that, upon CRISPR knocked, reduced PIKfyve-inhibitor-induced vacuole formation (Gayle et al., 2017). Given the potential role of $\mathrm{ClC}-7$ in lysosomal acidification, these results suggest that PIKfyve might regulate lysosomal $\mathrm{pH}$ by modulating $\mathrm{ClC}-7$ activity, and might thereby tune lysosomal traffic. Consistent with this hypothesis, $\mathrm{PI}(3,5) \mathrm{P} 2$ inhibits $\mathrm{ClC}-\mathrm{a}$, an $\mathrm{CLC}$ anion $/ \mathrm{H}^{+}$exchanger that contributes to the vacuolar acidification of plant cells, equivalent to lysosomes in animal cells (Carpaneto et al., 2017).

Here, using ratiometric measurements of lysosomal pH in cells treated with the PIKfyve inhibitor apilimod, we first explored the effects of PIKfyve inhibition on lysosomal pH and size. We then assessed the relationship between lysosomal $\mathrm{pH}$ and vacuole formation. Finally, we evaluated the role of $\mathrm{ClC}-7$ in this process, using both $\mathrm{pH}$ measurements of lysosomes from $\mathrm{ClC}-7$ knockout cells and patch-clamp electrophysiology of $\mathrm{ClC}-7$ trafficked to the plasma membrane. Moreover, we link these effects to a recently identified rare disease mutation, Y715C, that causes enlarged vacuoles and lysosomal hyperacidification, similar to the effects of apilimod, providing insight into the mechanism of $\mathrm{PI}(3,5) \mathrm{P} 2$ action on $\mathrm{ClC}-7$. Altogether, our data reveal that $\mathrm{PI}(3,5) \mathrm{P} 2$-inhibition of $\mathrm{ClC}-7$ provides a brake for the lysosomal acidification mechanism, preventing overacidification by the V-type ATPase. Our results further indicate that vacuole formation is not tightly coupled to lysosomal $\mathrm{pH}$ and that vacuoles can form even when normal lysosomal pH is perturbed. Finally, we demonstrate that the mechanisms of 
$\mathrm{PI}(3,5) \mathrm{P} 2$ inhibition of $\mathrm{ClC}-7$ and of the recently described disease-causing mutation $\mathrm{Y} 715 \mathrm{C}$ are tightly intertwined.

\section{RESULTS}

\section{Lysosomes swollen by PIKfyve inhibition are hyperacidic.}

We initially sought to determine the effect of PIKfyve inhibition on lysosomal pH using the Osteosarcoma Cell line U2OS; U2OS lysosomes are easily loaded with pH-sensing dyes and are distributed in the cells sparsely enough to image quantitatively and robustly. As our pH probe, we used Oregon Green 488-dextran (OG), a ratiometric pH sensitive dye with a pKa of 4.8, well suited for the lysosomal pH range and which is delivered effectively to lysosomes via fluid-phase endocytosis (DiCiccio and Steinberg, 2011).

We loaded endolysosomes and lysosomes, hereafter referred as lysosomes, with OG using standard methods with an overnight pulse of OG488-dextran followed by a 4-hr chase in dye-free media to allow the dye to accumulate in lysosomes. Most OG positive vesicles are lysosome-related compartments, as shown by staining with both Cathepsin-B and Lysotracker (Fig. 1 Supplement 1). After delivering OG fully to lysosomes, we treated cells for $3 \mathrm{~h}$ with $100 \mathrm{nM}$ of the PIKfyve inhibitor apilimod or its vehicle (0.25\% DMSO), enough to induce visible cytoplasmic vacuoles (Fig. 1, A and B). The OGcontaining vacuoles seen in these cells are enlarged lysosomes (Bissig et al., 2017; Choy et al., 2018; Sharma et al., 2019). We measured lysosomal pH using excitation-ratio imaging, including full pH calibration curves in every experiment. Though apilimod causes dramatic changes in lysosome size, overlap between $\mathrm{pH}$ calibration curves in control and apilimod treated conditions shows that vacuole properties don't interfere with $\mathrm{pH}$ measurement (Fig. 1C). A representative experiment reveals a substantial shift in lysosomal pH in cells treated with apilimod compared with control (Fig 1D), further supported by averaged results from multiple independent experiments (Fig 1F). In control conditions the lysosomal $\mathrm{pH}$ is $4.32 \pm 0.04$ (mean $\pm \mathrm{SEM}$ ). We find that, in addition to its well-known effects on size, apilimod treatment induces robust lysosomal hyperacidification, reaching an average $\mathrm{pH}$ of $4.02 \pm 0.05$. This decrease of $0.31 \mathrm{pH}$ unit corresponds to a doubling of the free proton concentration of the lysosomal lumen compared to control (Fig. 1F). The global shift of the cumulative distribution of individual lysosomal pHs reveals that apilimod-induced lysosomal hyperacidification is homogeneous and not restricted to a subset of lysosomes (Fig. 1E). Similar measurements in neonatal fibroblasts and SUDHL B-cell lymphoma cells confirmed that their lysosomes also hyperacidify when treated with apilimod (Fig 1 Supplement 2). Taken together, these results establish that acute pharmacological 
inhibition of PIKfyve indeed affects lysosomal pH, surprisingly causing hyperacidification. Welldocumented hyperacidification is rare in lysosomal manipulation or disease, though we recently reported a disease-causing CIC-7 gain-of-function mutation with this effect (Nicoli et al., 2019).

A

\begin{tabular}{|c|c|c|}
\hline OG loading & Chase & 7 \\
\hline & & \\
\hline
\end{tabular}

B

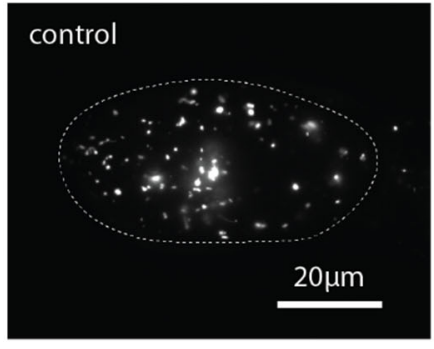

D

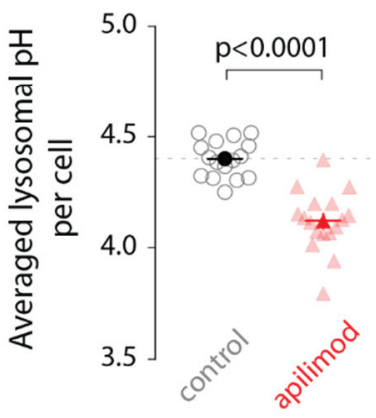

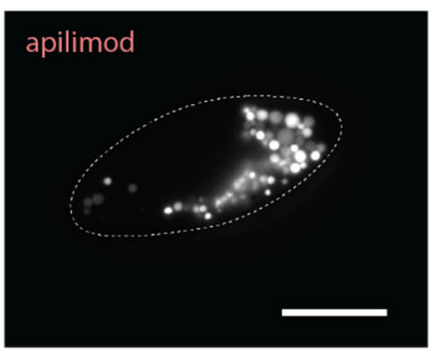

$\mathrm{E}$

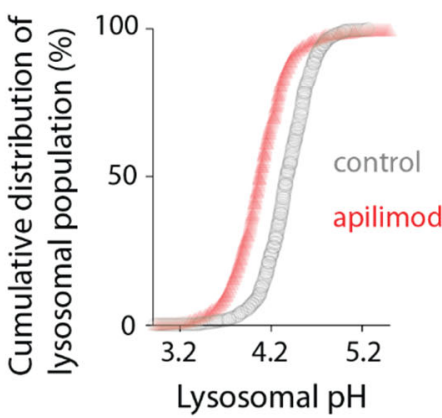

C

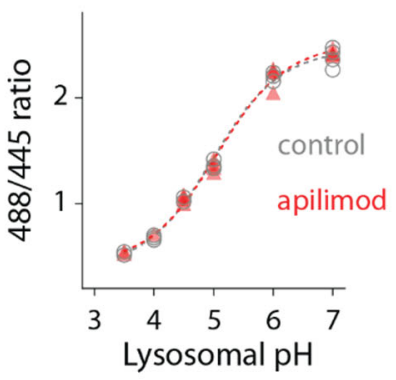

$\mathrm{F}$

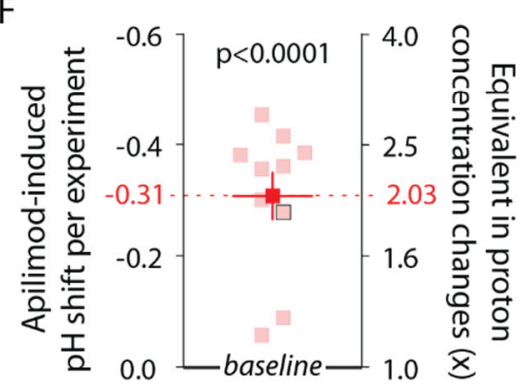

FIGURE 1: Lysosomes swollen by PIKfyve inhibition are hyperacidic. (A) Protocol timeline. U2OS cells were "Iysosomeloaded" with Oregon Green 488 dextran (OG loading) and treated for 3h with PIKfyve inhibitor apilimod (100nM, red) or its vehicle (0.25\% DMSO, control) before imaging. (B - E) A representative experiment. (B) Images of cells acquired by $445 \mathrm{~nm}$ laser excitation. Bright objects represent OG positive lysosomes in control (left) versus apilimod (right) conditions. Dotted lines delineate cell outlines. (C) pH calibration curves obtained in control (grey empty circle) or apilimod (red triangle) conditions. Each symbol represents the averaged lysosomal 488/445 ratio of one cell (4 cells per condition). (D) Lysosomal pH measured in control (left, grey empty circles, 16 cells, $4.40 \pm 0.02$ ) versus $100 \mu \mathrm{M}$ apilimod (red triangles, 17 cells, $4.12 \pm 0.03$ ) for an individual experiment. Dark symbols represent averages over all cells in the experiment. Each pale symbol represents the averaged lysosomal $\mathrm{pH}$ of one cell. Unpaired t-test: $\mathrm{p}<0.0001$. (E) Cumulative distribution of individual lysosomal $\mathrm{pH}$ for control (grey empty circle, 16 cells, 732 lysosomes) versus apilimod (red triangle, 17 cells, 628 lysosomes) conditions from the same experiment as in Figure 1D. Each symbol represents the fraction of lysosomes having a $\mathrm{pH}$ value below that represented in the abscissa. (F) Apilimod-induced pH shifts $(-0.31 \pm 0.04)$ from multiple independent experiments and corresponding fold changes in proton concentration ( $2.03 \pm 0.17) ; 10$ experiments (8-17 cells per condition in each experiment). Proton concentration change $\left(\left[\mathrm{H}^{+}\right]_{\text {change }}\right)$ was calculated from apilimod-induced $\mathrm{pH}$ shift $(\Delta \mathrm{pH})$ using the following relation: $\left[\mathbf{H}^{+}\right]_{\mathbf{c h a n g e}}=\mathbf{1 0}^{-\Delta \mathbf{p H}}$. Paired t-test: $p<0,0001$. Dark symbols represent averages over all independent experiments. Each pale symbol represents the averaged lysosomal pH from multiple cells in one experiment. The dot delineated by a black box corresponds to the experiment presented in panels B-E. Data are displayed as mean \pm SEM.

\section{Lysosomal swelling and hyperacidification are independent events}

In cultured cells, pharmaceutical inhibition of PIKfyve depletes PI(3,5)P2 on the time scale of minutes (Zolov et al., 2012). Subsequently, lysosomes increase in size steadily over time (Choy et al., 2018), an effect that reverses over several hours after drug removal (Choy et al., 2018; Bissig et al., 
2017). We analyzed the time course of PIKfyve induced lysosomal swelling and hyperacidification to investigate the relationship between the two effects.

Measuring lysosomal pH and size in OG-loaded U2OS cells treated with 100nM apilimod for $30 \mathrm{~min}, 1 \mathrm{~h}, 3 \mathrm{~h}$ and $24 \mathrm{~h}$, revealed that, as previously reported, initial appearance of vacuoles is rapid (Fig. 2, A, B, and D) (Choy et al., 2018). However, whereas lysosome hyperacidification is complete (stabilizing at $0.27+/-0.05 \mathrm{pH}$ below control) during the first hour of treatment, the organelles continue enlarging over the next 24h, (Fig. 2C). Treatment with another recently identified PIKfyve inhibitor, WX8 $(1 \mu \mathrm{M})($ Sharma et al., 2019), recapitulates the effects of apilimod, ruling out an off-target effect (Fig. 2E). Plotting $\mathrm{pH}$ vs size of individual lysosomes during hyperacidification (30min and $1 \mathrm{~h}$ ) revealed no detectable correlation (Fig. 2 Supplement 1). After apilimod removal lysosomes quickly begin to shrink via tubulation events (Fig. 2F, G orange box and Fig. 2l) but lysosomal pH starts to recover only after $2 \mathrm{~h}$ (when lysosomal size is almost back to normal Fig. 2, $\mathrm{H}$ and $\mathrm{I}$ ), fully recovering more than $6 \mathrm{~h}$ after apilimod washout (Fig. 2H).

Thus, both the kinetics and extent of the changes in lysosomal size and pH during and after apilimod treatment are quite distinct, suggesting that the processes underlying these changes are at least partially independent. 
A

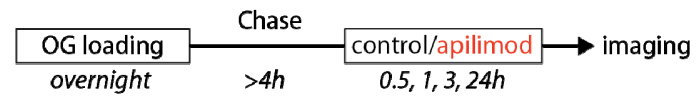

B
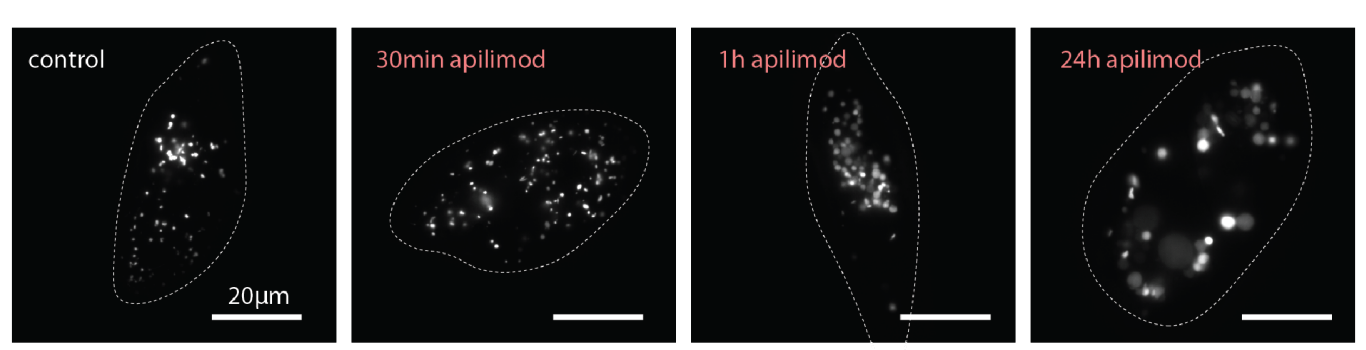

C
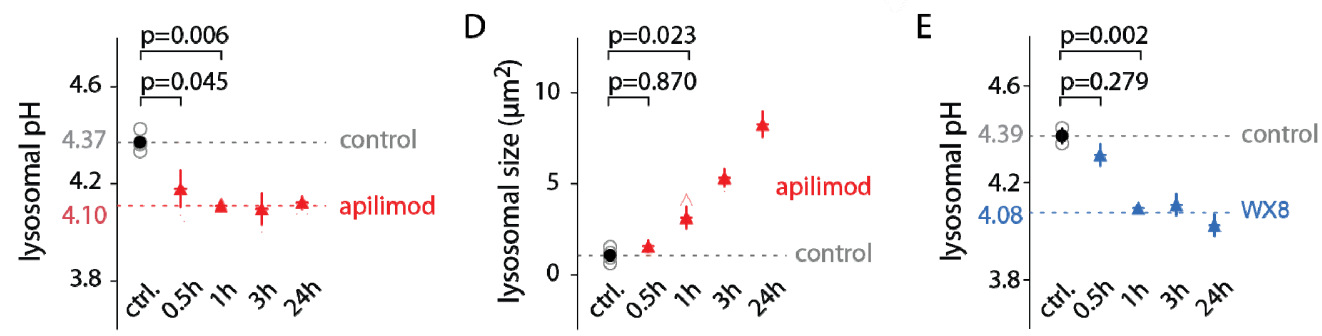

Apilimod treatment time

Apilimod treatment time

WX8 treatment time

$\mathrm{F}$

\begin{tabular}{|c|c|c|c|}
\hline & Chase & & Recovery \\
\hline $\begin{array}{c}\text { OG loading } \\
\text { overnight }\end{array}$ & & $\frac{\text { control/apilimod }}{2 h}$ & $0.5,1,2,3,6,24 h$ \\
\hline
\end{tabular}

G
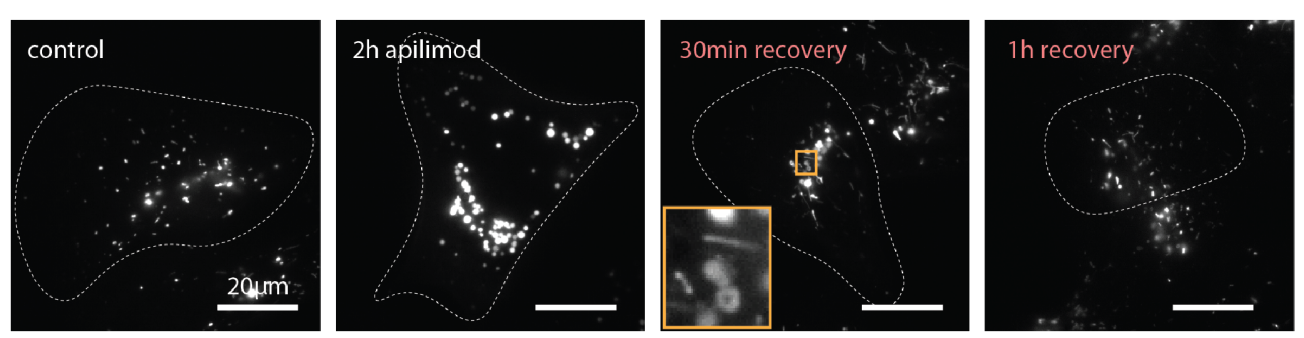

$\mathrm{H}$
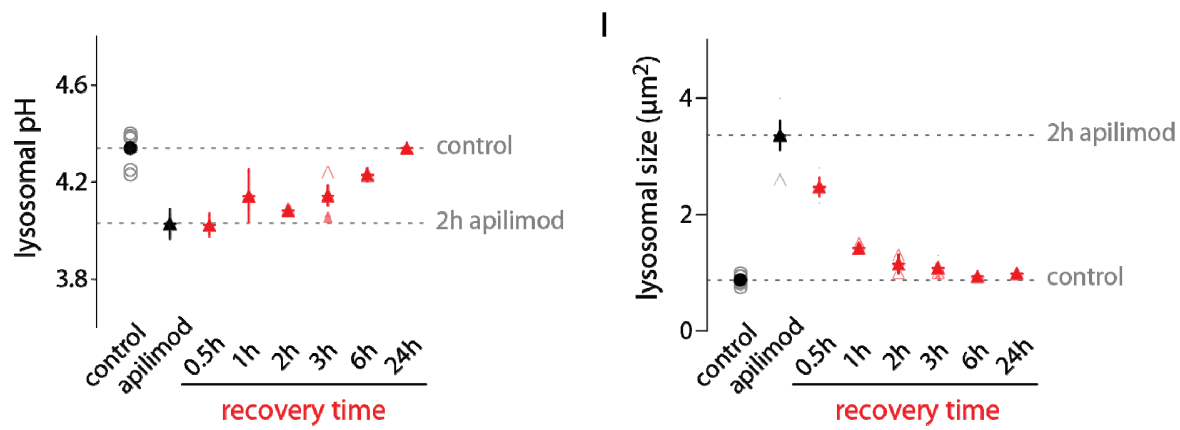

FIGURE 2: Evolution of lysosomal pH and size during PIKfyve inhibition and recovery. (A) Protocol timeline to analyze the evolution of lysosomal pH and size during PIKfyve inhibition. U2OS cells were "lysosome-loaded" with OG then treated for $30 \mathrm{~min}, 1 \mathrm{~h}, 3 \mathrm{~h}$ or $24 \mathrm{~h}$ with apilimod $(100 \mathrm{nM}$, red) or its vehicle $(0.25 \%$ DMSO, control) before imaging. (B)

Representative images of cells acquired by $445 \mathrm{~nm}$ laser excitation. Bright objects represent OG positive lysosomes. Dotted lines delineate cell outlines. (C - D) Comparison of lysosomal pH (C) and size (D) in control (grey empty circle) and after different apilimod treatment times (red triangle). Each symbol represents the averaged lysosomal pH of one experiment (4 independent experiments, $10-15$ cells per condition per experiment). Red dashed line in (C) represents the average $\mathrm{pH}$ value of $1 \mathrm{~h}$ to $24 \mathrm{~h}$ apilimod time points. P-values are obtained from one-way ANOVA Dunnett's multiple comparisons test. (E) Comparison of lysosomal $\mathrm{pH}$ in control condition (grey empty circle) and after different treatment times with the PIKfyve inhibitor WX8 (1 $\mu \mathrm{M}$, blue triangle). Each symbol represents the averaged lysosomal pH of one experiment (2 experiments, 10-13 cells per condition 
per experiment). Blue dashed line represents the average value of $1 \mathrm{~h}$ to $24 \mathrm{~h} W X 8$ time points. (F) Protocol timeline to analyze the recovery of lysosomal $\mathrm{pH}$ and size after washout of apilimod. U2OS cells were "Iysosome-loaded" with OG, treated $2 \mathrm{~h}$ with $100 \mathrm{nM}$ apilimod or its vehicle $(0.25 \% \mathrm{DMSO}$, control) to induce lysosomal hyperacidification and swelling. Apilimod was subsequently washed out with fresh media and imaged $30 \mathrm{~min}, 1 \mathrm{~h}, 3 \mathrm{~h}, 6 \mathrm{~h}$ and $24 \mathrm{~h}$ after the washout. (G) Representative images of cells acquired using $445 \mathrm{~nm}$ laser excitation. Bright objects represent OG positive lysosomes. Dotted lines delineate cell outlines. The orange box at $30 \mathrm{~min}$ time point highlights lysosomal tubulation events. $\mathbf{H}-\mathbf{I})$ Comparison of lysosomal $\mathrm{pH}(\mathbf{H})$ and size (I) in control condition (grey empty circle), after $2 \mathrm{~h}$ apilimod treatment (black triangle) and at different times after apilimod washout (red triangle). Each symbol represents the average lysosomal pH of one experiment (3-7 experiments, 8-15 cells per condition per experiment).

\section{Lysosomal hyperacidification is not required for vacuole formation.}

Our results regarding the kinetics of apilimod-induced lysosomal $\mathrm{pH}$ and size changes suggest that these two effects may occur through different mechanisms (Fig. $2 \mathrm{H}$ and I). To test this possibility, we evaluated whether alkalinizing lysosomal compartments could prevent vacuole formation. This question was previously addressed in part using the V-ATPase inhibitor BafA1 and the lysosomotrophic agents chloroquine and $\mathrm{NH}_{4} \mathrm{Cl}$ in the monkey fibroblast COS7 cell line (Compton et al., 2016). Although V-ATPase inhibition in that study clearly prevents vacuole formation, the $\mathrm{pH}$ modifying reagents $\mathrm{NH}_{4} \mathrm{Cl}$ and chloroquine used in that paper were added 40 minutes after pharmacological inhibition of PIKfyve; given our results, this changes the $\mathrm{pH}$ well after the development of lysosomal hyperacidification and probably too late to affect early $\mathrm{pH}$-dependent processes (Fig. 2C).

To determine the influence of lysosomal pH early in the process of apilimod-induced enlargement, we manipulated the $\mathrm{pH}$ by adding either $12 \mu \mathrm{M}$ of chloroquine, $100 \mathrm{nM}$ of BafA1, or a vehicle (water/DMSO) to OG-loaded U2OS cells 30min prior to and throughout $3 \mathrm{~h} 100 \mathrm{nM}$ apilimod treatment (Fig. 3A), then imaged cells to quantify lysosomal pH and size (Fig. 3B). 30min treatment with $12 \mu \mathrm{M}$ chloroquine is long enough to alkalinize lysosomes to $\sim \mathrm{pH} 5.5-6.0$ (Fig. $3 \mathrm{C}$ and Fig. 3 Supplement 1), a range where $\mathrm{OG}$ is still sensitive to $\mathrm{pH}$ variations (Fig. 1C). As previously reported, inhibition of VATPase with BafA1 prevents vacuole formation (Compton et al., 2016; Sharma et al., 2019) (Fig. 3D). Conversely, vacuoles still form with chloroquine pre-treatment (Fig. 3D). To distinguish the effect of pH from that of direct V-ATPase inhibition, we added $25 \mu \mathrm{M}$ chloroquine to raise the lysosomal pH to above the value resulting from Bafilomycin treatment. We still observed the same level of vacuolization upon apilimod treatment, ruling out the $\mathrm{pH}$ change due to V-ATPase inhibition as the cause (data not shown). Interestingly, even after alkalinization with chloroquine, apilimod exposure significantly lowered lysosomal pH (Fig. 3C; $5.63 \pm 0.13$ SEM (untreated) versus $5.22 \pm 0.10$ SEM (apilimod); Paired t-test, $p=0.02$ ). Both hyperacidification and vacuole formation are entirely lost when the V-ATPase is inhibited

(Fig. 3C; $6.26 \pm 0.20$ (untreated) vs $6.25 \pm 0.23$ (Apilimod)), although OG is poorly sensitive to pH changes at this almost neutral pH (Fig. 1C). Taken together, these results prove that the early hyperacidification 
of lysosomes induced by PIKfyve inhibition is not required for vacuole formation but confirm that VATPase activity is necessary for the process.

A

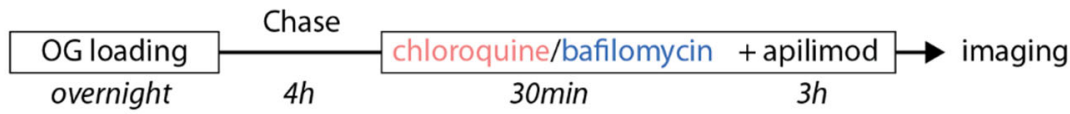

B
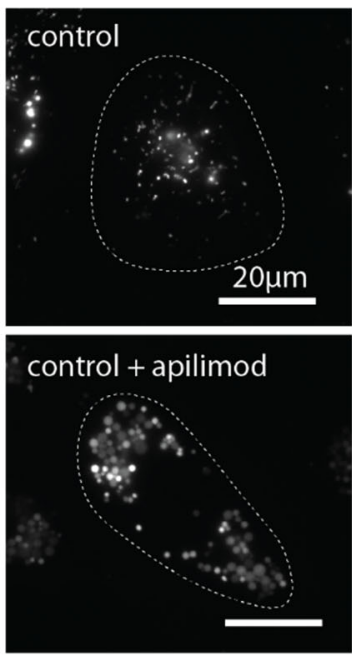

C

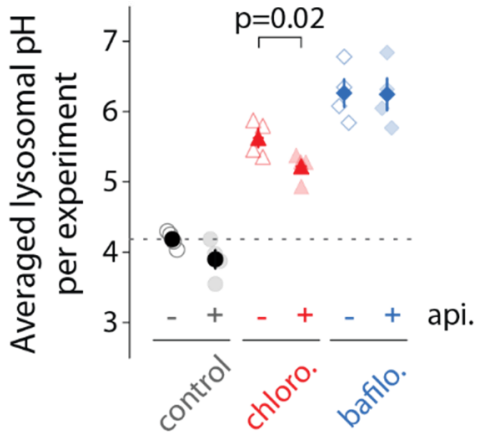

\section{chloroquine}

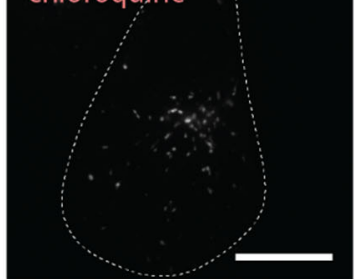

chloroquine + apilimod

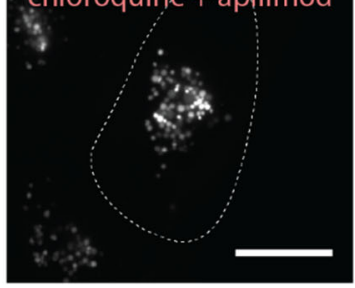

$\mathrm{D}$

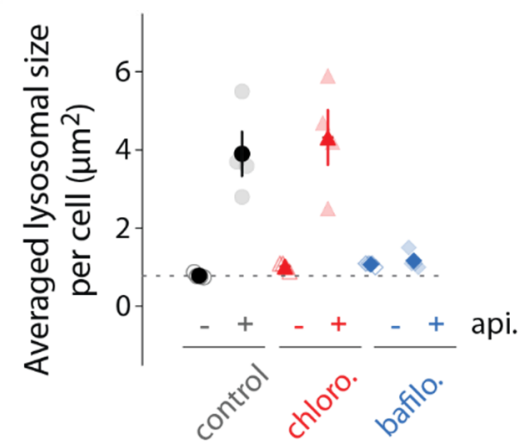

FIGURE 3: Alkilinazing lysosomal pH does not prevent lysosomal swelling. (A) Protocol timeline. U2OS cells were "Iysosomeloaded" with OG and pre-treated 30min with chloroquine $(12 \mu \mathrm{M}$, red), BafA1 (100nM, blue) or a vehicle $(0.31 \%$ DMSO, grey), before $3 \mathrm{~h}$ treatment with apilimod (100nM, bottom images) or a vehicle (control, DMSO $0.31 \%$, upper images). (B) Representative images of cells acquired by $445 \mathrm{~nm}$ laser excitation. Bright objects represent $\mathrm{OG}$ positive lysosomes. Dotted lines delineate cells outlines. (C, D) Comparison of lysosomal pH (C) or size (D) in control (grey), chloroquine (chloro., red) and BafA1 (bafilo., blue) conditions during untreated (api. -, empty symbols) versus apilimod treated (api. +, filled symbols) conditions. Each symbol represents the averaged lysosomal pH or size of one experiment (4 experiments; 8-11 cells per condition). In (C) Apilimod induces a lysosomal $\mathrm{pH}$ shift to more acidic value in chloroquine condition (Paired $\mathrm{t}$-test, $\mathrm{p}=0.02$ ).

\section{Increasing $\mathrm{PI}(3,5) \mathrm{P} 2$ levels does not affect lysosomal pH}

PIKfyve inhibition, and consequent PI(3,5)P2 depletion, hyperacidifies lysosomes to a new, lower, pH set-point (Fig. 2C). We also tested whether increasing PI(3,5)P2 levels influenced lysosomal pH using a hyperactive mutant form of PIKfyve (PIKfyve ${ }^{\mathrm{KYA}}$ ) which raises PI(3,5)P2 levels by several fold in mouse hippocampal cultured neurons when overexpressed (McCartney et al., 2014b). As a negative 
control we overexpressed WT PIKfyve (which does not change PI(3,5)P2 levels) (McCartney et al., 2014b).

We measured lysosomal pH of OG-loaded U2OS cells $24 \mathrm{~h}$ after transfection with either PIKfyve ${ }^{\mathrm{KYA}}$ or PIKfyve (Fig. 4A, B). Because both PIKfyve versions were untagged, we used cotransfected mCherry as a marker for transfection (Fig. 4C); since co-transfected plasmids usually are delivered to the same cells, the mCherry positive population must also be enriched in cells expressing the PIKfyve constructs. Note that mCherry expression doesn't impact lysosomal pH (Fig 4D). Cells coexpressing mCherry and PIKfyve ${ }^{\mathrm{KYA}}$ show similar lysosomal pH and size compared to controls (Fig. 4, D and E). Thus, while lowering PI(3,5)P2 results in a pH change, raising it does not. Perhaps the effector(s) downstream of the lipid in the pathway that affects $\mathrm{pH}$ is already saturated with the lipid under normal cellular conditions in U2OS cells.

A

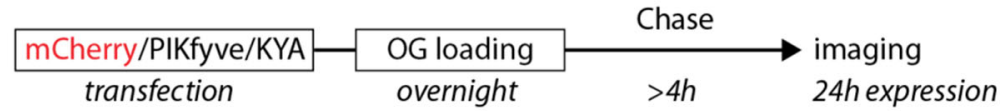

B

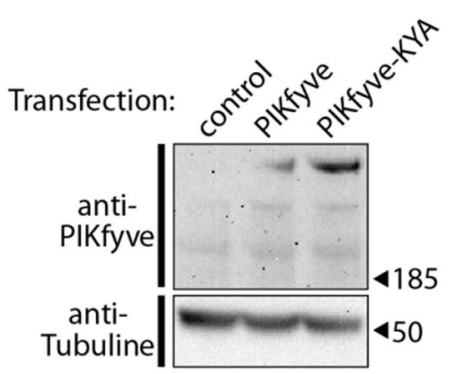

C
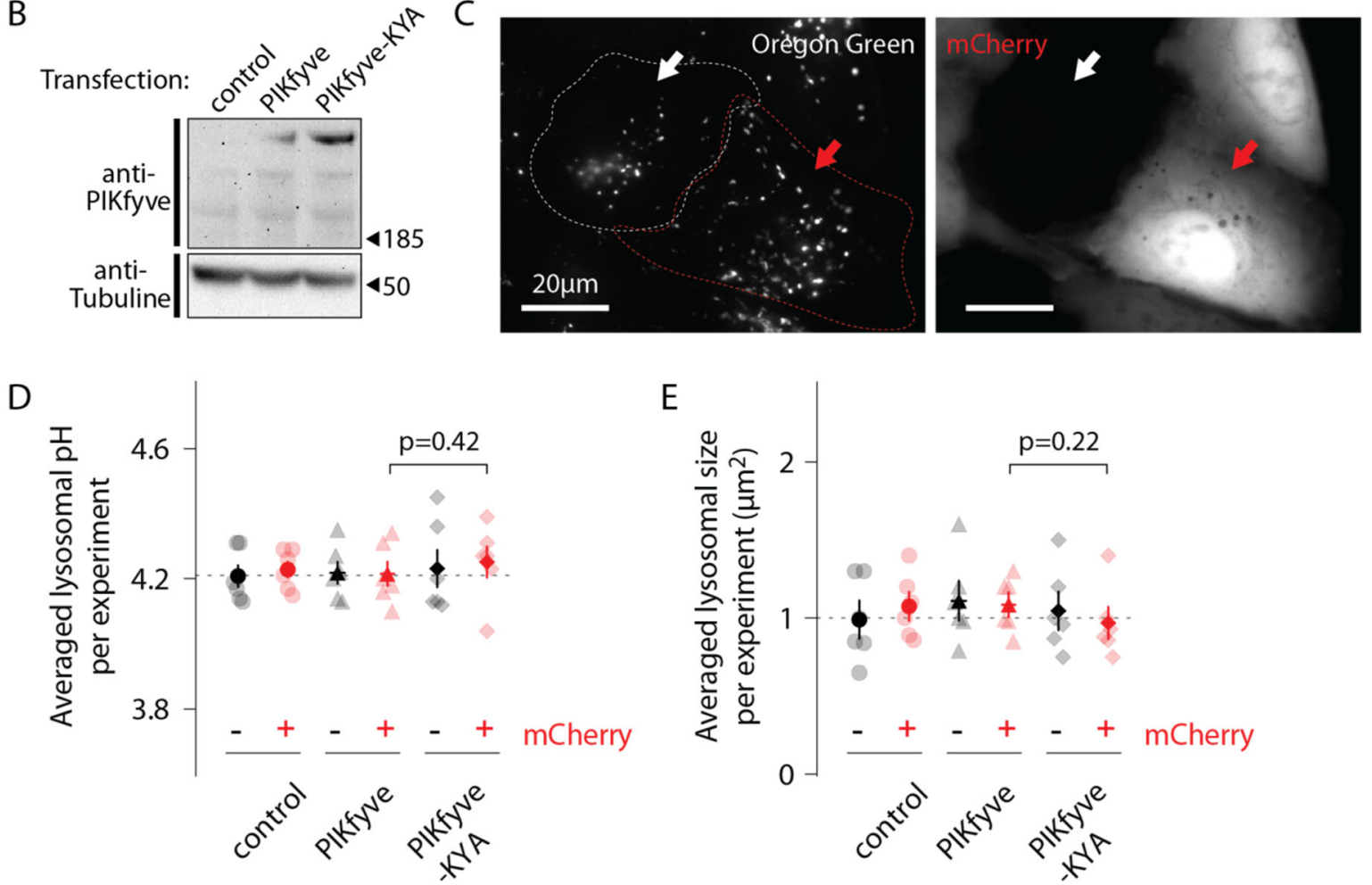

FIGURE 4: Increasing PI(3,5)P2 levels does not affect lysosomal pH. (A) Protocol timeline. U2OS cells were transfected with either mCherry alone, mCherry and PIKfyve, or mCherry and PIKfyve ${ }^{\mathrm{KYA}}$ and subsequently "lysosome-loaded" with OG. Cells were imaged after $24 \mathrm{~h}$ expression to quantify lysosomal $\mathrm{pH}$ and size. (B) Western-blot indicating PIKfyve or PIKfyve ${ }^{\mathrm{KYA}}$ expression level for each condition after 24h expression. Note that PIKfyve endogenous level (control) was too low to be detected. (C) Representative cells imaged by $445 \mathrm{~nm}$ laser excitation (OG channel, left image) and 640nm laser excitation (mCherry channel, right image). The red arrow indicates one cell containing lysosomes loaded with OG (bright dots in OG channel) and expressing mCherry (bright cell in mCherry channel). The white arrow indicates one cell from the same batch 
bioRxiv preprint doi: https://doi.org/10.1101/2021.10.07.463477; this version posted October 7, 2021. The copyright holder for this preprint (which was not certified by peer review) is the author/funder. This article is a US Government work. It is not subject to copyright under 17 USC 105 and is also made available for use under a CCO license.

containing lysosomes filled with OG but not expressing mCherry. Dotted lines delineate cell shape. (D, E) Comparison of lysosomal pH (D) or size (E) in control (circle), PIKfyve-transfected (triangle) and PIKfyve ${ }^{\mathrm{KYA}}$-transfected (diamond) conditions. For each condition, cells were separated into two populations based on the presence or absence of mCherry. Each symbol represents the averaged lysosomal $\mathrm{pH}$ or size of one experiment ( 6 experiments; 8-11 cells per condition per experiment). Pvalues: Paired t-test between PIKfyve-mCherry and PIKfyve ${ }^{\mathrm{KYA}}$-mCherry conditions. 


\section{CIC-7 is involved in both lysosomal hyperacidification and size regulation}

Recently, Gayle et al. observed that the loss of either CIC-7 or its obligate $\beta$-subunit, Ostm1, provides resistance against vacuole formation induced by apilimod treatment in B-cell Non Hodgkin Lymphoma (Gayle et al., 2017). Since CIC-7 has been hypothesized to facilitate lysosomal acidification (Graves et al., 2008; Ishida et al., 2013; Mindell, 2012), and as apilimod shifts lysosomal pH to a new set point (Fig. 2C), we analyzed the impact of CLCN7 knock out on PIKfyve inhibition, generating a U2OS CLCN7 knock out cell line (CIC-7 KO) using CRISPR/Cas9 (Shalem et al., 2014). Without good available antibodies for CIC-7, we validated CLCN7 allele deletion both at genomic and transcript levels (Fig. 5A and Fig. 5 Supplement 1). This ClC-7 KO cell line has two different CLCN7 alleles with 64bp or 118bp frameshift deletions in EXON1 (Fig. 5B). No wild type allele remains.

To probe for effects of knocking out ClC-7 on apilimod sensitivity, we measured lysosomal pH in WT and CIC-7 KO U2OS cells (100 nM apilimod, 3h; Fig. 5C, D). Overlap of both pH calibration curves and cumulative distribution plots of individual lysosomal $\mathrm{pH}$ for WT and KO lysosomes demonstrates that knocking out ClC-7 affects neither lysosomal OG loading nor trans-membrane pH equilibration (Fig. 5 Supplement 2). Consistent with previous reports for several cell types in primary culture (Kasper et al., 2005; Lange et al., 2006; Weinert et al., 2010), knocking out CIC-7 in untreated U2OS cells doesn't alter lysosomal pH (Fig. 5G). The size of untreated CLC-7 KO lysosomes is also unchanged from WT (Fig. 5F). Strikingly, however, knocking out ClC-7 strongly diminishes apilimod-induced lysosomal hyperacidification: the $\Delta \mathrm{pH}$ is only about 0.13 units in CLC-7 KO cells compared to 0.31 units in WT cells (Fig. 5, representative experiment in E; average of multiple independent experiments in $\mathrm{H} ; 0.31 \mathrm{pH}$ units \pm 0.04 SEM, WT; versus 0.13 pH units \pm 0.04 SEM, ClC-7; P=0.0119). Similarly, lysosome swelling still occurs upon apilimod treatment in ClC-7 KO cells, but to a lesser extent (Fig. 5, representative experiment in F; average of multiple independent experiments in I; $4.11 \mu \mathrm{m}^{2} \pm 0.37$ SEM versus 2.04 $\mu \mathrm{m}^{2} \pm 0.29$ SEM for CLC-7 WT and KO cells, respectively; $\mathrm{P}=0.0017$ ).

Overall, these results indicate that $\mathrm{ClC}-7$ is involved in two important mechanisms downstream of PIKfyve. First, it contributes to setting the lysosomal pH, at least under some circumstances. Second, CIC-7 participates in the regulation of lysosomal size and/or lysosomal trafficking through a yet unknown chanism. 
bioRxiv preprint doi: https://doi.org/10.1101/2021.10.07.463477; this version posted October 7, 2021. The copyright holder for this preprint (which was not certified by peer review) is the author/funder. This article is a US Government work. It is not subject to copyright under 17 USC 105 and is also made available for use under a CCO license.

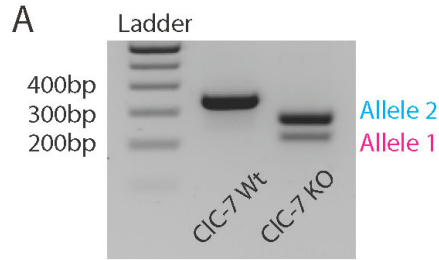

B

ATGGCCAACGTCTCTAAGAAGGTGTCCTGGTCCGGCCGGGACCGGGACGACGAGGAGGCGGCGCCGCTGCTG CGGAGGACGGCGCGGCCCGGCGGGGGGACGCCGCTGCTGAACGGGGCTGGGCCCGGGGCTGCGCGCCAG

CLCN7 EXON 1 sequence

Allele 1 deletion site (118bp with frameshift)

Allele 2 deletion site (64bp with frameshift)

C

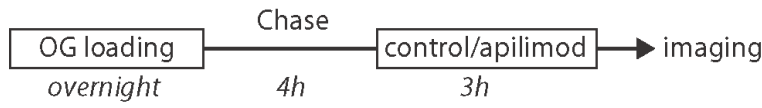

D

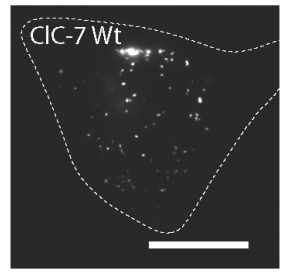

CIC-7 Wt + apilimod

8* $\cdots$

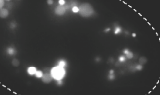

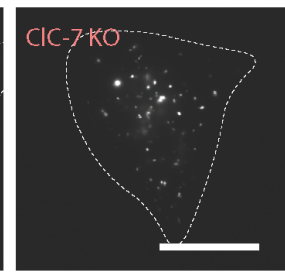

$\mathrm{CIC}-7 \mathrm{KO}$, * apìilimod

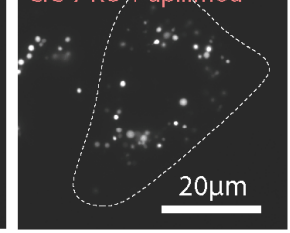

$\mathrm{E}$

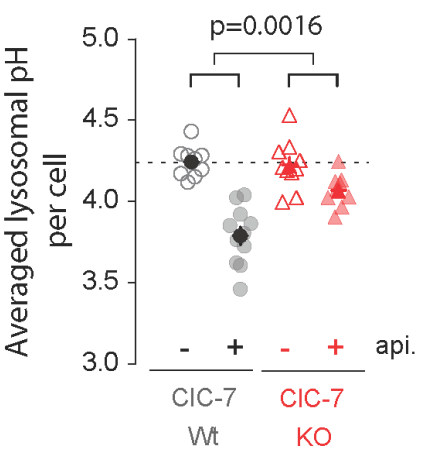

G

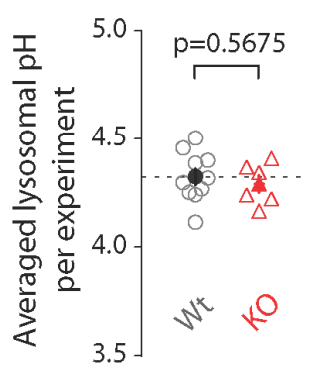

$\mathrm{H}$

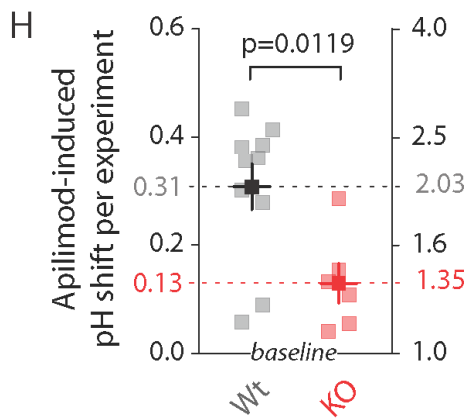

F

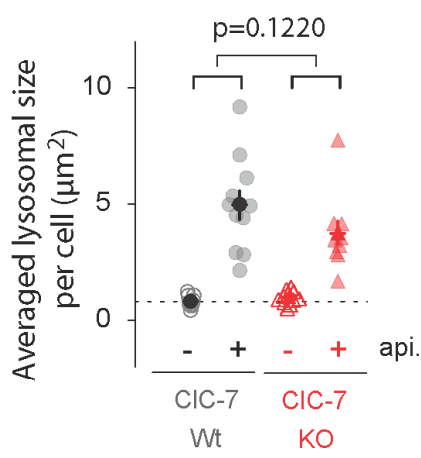

FIGURE 5: CIC-7 KO cells display both lysosomal hyperacidification and swelling reduction. (A) Agarose gel electrophoresis of PCR product amplified from CICN7 deletion site of wild type (CIC7 Wt, left) and CLCN7 knock-out (CIC-7 KO, right) U2OS cells. (B) EXON1 sequence from CLCN7 WT (black) and KO (purple and teal) alleles. (C) Protocol timeline. Cells were "lysosome-loaded" with OG and treated for $3 \mathrm{~h}$ with apilimod $(100 \mathrm{nM}$, red) or its vehicle $(0.25 \%$ DMSO, control) before imaging. (D) Images from a representative experiment: ClC-7 WT (left) or CIC-7 KO (right) cells acquired by 445nm laser excitation. Bright objects represent OG positive lysosomes in control (top) versus apilimod (bottom) conditions. Dotted lines delineate cell outlines. (E, F) Lysosomal pH (E) or size (F) from CIC-7 WT (grey circle) or ClC-7 KO (red triangle) cells in apilimod (filled symbols) versus control (empty symbols) in a representative experiment. Dark symbols are averages over all cells; each pale symbol represents the average lysosomal $\mathrm{pH}$ from one cell. P-values for apilimod effects are obtained from two-way ANOVA. (G) There is no significant difference in $\mathrm{pH}$ between untreated WT and untreated $K O$ cells ( $P=0.5675$, unpaired t-test). For $G, H, l$, dark symbols are averages over all experiments; each pale symbol represents the averaged lysosomal $\mathrm{pH}$ or size from one experiment (10 and 6 independent experiments for WT and KO conditions, respectively; each experiment represents 8-18 cells per condition). (H,I) Comparison of lysosomal pH-shift $(\mathbf{H})$ or size-shift $(\mathbf{I})$ induced by apilimod treatment in $\mathrm{ClC}-7 \mathrm{Wt}$ (grey) 
versus $\mathrm{ClC}-7 \mathrm{KO}$ (red) cells. . Proton concentration change $\left(\mathbf{H},\left[\mathrm{H}^{+}\right]_{\text {change }}\right)$ was calculated from apilimod-induced $\mathrm{pH}$ shift $(\Delta \mathrm{pH})$ using the following relation: $\left[\mathrm{H}^{+}\right]_{\text {change }}=10^{-\Delta \mathrm{pH}}$. P-values from unpaired t-test.

\section{PI(3,5)P2 inhibits ClC-7 transporter currents}

In the simplest model for our observations, the effect of PI(3,5)P2 on ClC-7 would be via a direct interaction with the protein, thereby modulating its activity and its contribution to lysosomal acidification. Our results so far are consistent with a hypothesis in which PI(3,5)P2 inhibits ClC-7 transport activity: relief of this inhibition, as by $\mathrm{PI}(3,5) \mathrm{P} 2$ removal, would activate $\mathrm{ClC}-7$, allow excess $\mathrm{Cl}^{-}$ to enter the lysosome, and thereby provide excess counterions to facilitate further (and excessive) acidification by the V-type ATPase. A recently published cryo-EM structure of the human CIC-7/Ostm1 complex demonstrates that a direct interaction is indeed possible, revealing electron density compatible with a phosphoinositide bound to the protein (Fig. 6A, B) (Schrecker et al., 2020). With the head group located at the interface between the cytosolic and transmembrane domains (Schrecker et al., 2020), the positioning of the bound lipid is intriguing, especially given that a large body of evidence that CLC cytoplasmic domains play important roles in the slow voltage-dependent gating observed in these proteins (Bykova et al., 2006; Ludwig et al., 2013). In this context, we next sought to directly test for a functional effect of $\mathrm{PI}(3,5) \mathrm{P} 2$ on $\mathrm{ClC}-7$ activity by measuring transport currents using patch clamp electrophysiology. We transfected HEK-293 cells with a ClC-7 construct containing mutations to the Nterminal lysosome-targeting motif that enable functional transporters to be trafficked to the plasma membrane (CIC-7PM), allowing measurement of whole-cell currents using patch clamp (Leisle et al., 2011; Stauber and Jentsch, 2010). In lysosomes, PI(3,5)P2 is found in the cytosolic leaflet of the membrane; thus, to introduce the phospholipid, we added $50 \mu \mathrm{M}$ short-chain $\mathrm{PI}(3,5) \mathrm{P} 2$ to the pipette solution, a concentration that theoretically would result in 1 mol\% PI(3,5)P2 in the cytosolic membrane (Collins and Gordon, 2013). We expected that after initial break-in, the lipid would gradually diffuse into the cell and partition into the membrane over the span of a few minutes.

We obtained a family of voltage-dependent CIC-7PM currents immediately upon break-in, before the PIP2 had time to enter the cell. After waiting three minutes for the PIP2 to diffuse into the cytosol, we recorded a second voltage family in the presence of the lipid. The ratios of these measurements reflect the effect of the signaling lipid on the transporter current. Recordings made without PI(3,5)P2 in the pipette showed little change between the two timepoints; activation and deactivation kinetics and current magnitudes remained constant, demonstrating that the $\mathrm{ClC}-7$ currents are stable over time. However, including PI(3,5)P2 in the pipette resulted in a $~ 39 \%$ decrease in maximum voltage-activated currents and $66 \%$ decrease in the amplitude of tail currents following 
repolarization (Fig 6C, E). Increasing the time for the lipid to diffuse into the cell by increasing the time until the second recording to 7 minutes after break-in did not result in further inhibition (data not shown). The deactivation rate was also significantly faster in the presence of PI(3,5)P2 (Fig. 6 Supplement $1 \mathrm{~A})$. The effects of PI(3,5)P2 on maximal current and tail current amplitude were dosedependent with increasing inhibition by PI(3,5)P2 over concentrations between 25-100 $\mu \mathrm{M}$ (Fig 6 Supplement 1E). PI(3,5)P2-inhibition of CIC-7 appears to be specific: neither PI3P or PI(4,5)P2 yielded detectable inhibition of the ClC-7 currents (Fig 6G, H, Fig 6 Supplement 1C, D). Because the lipid bound to $\mathrm{ClC}-7$ in the structure was modeled as a PI3P (due to steric clashes between the 5-phosphate and adjacent residues when PI(3,5)P2 was modeled), its lack of effect on CIC-7 currents was surprising. Since the lipid in the CryoEM structure was not added during the purification, it must have been carried through the preparation bound to $\mathrm{ClC}-7$, and should be tightly bound, but the lack of effect in our experiments suggests, at best, a low affinity. To further test for an interaction with PI3P, we therefore attempted competition experiments with equimolar mixes of $\mathrm{PI}(3,5) 2$ and $\mathrm{PI} 3 \mathrm{P}$; the presence of $\mathrm{PI} 3 \mathrm{P}$ in these experiments did not detectably affect the inhibition by PI(3,5)P2, suggesting a specific effect of the doubly phosphorylated lipid (Fig 6G, H, Fig 6 Supplement 1C, D). We conclude that PI(3,5)P2 specifically inhibits ClC-7 transport currents. 


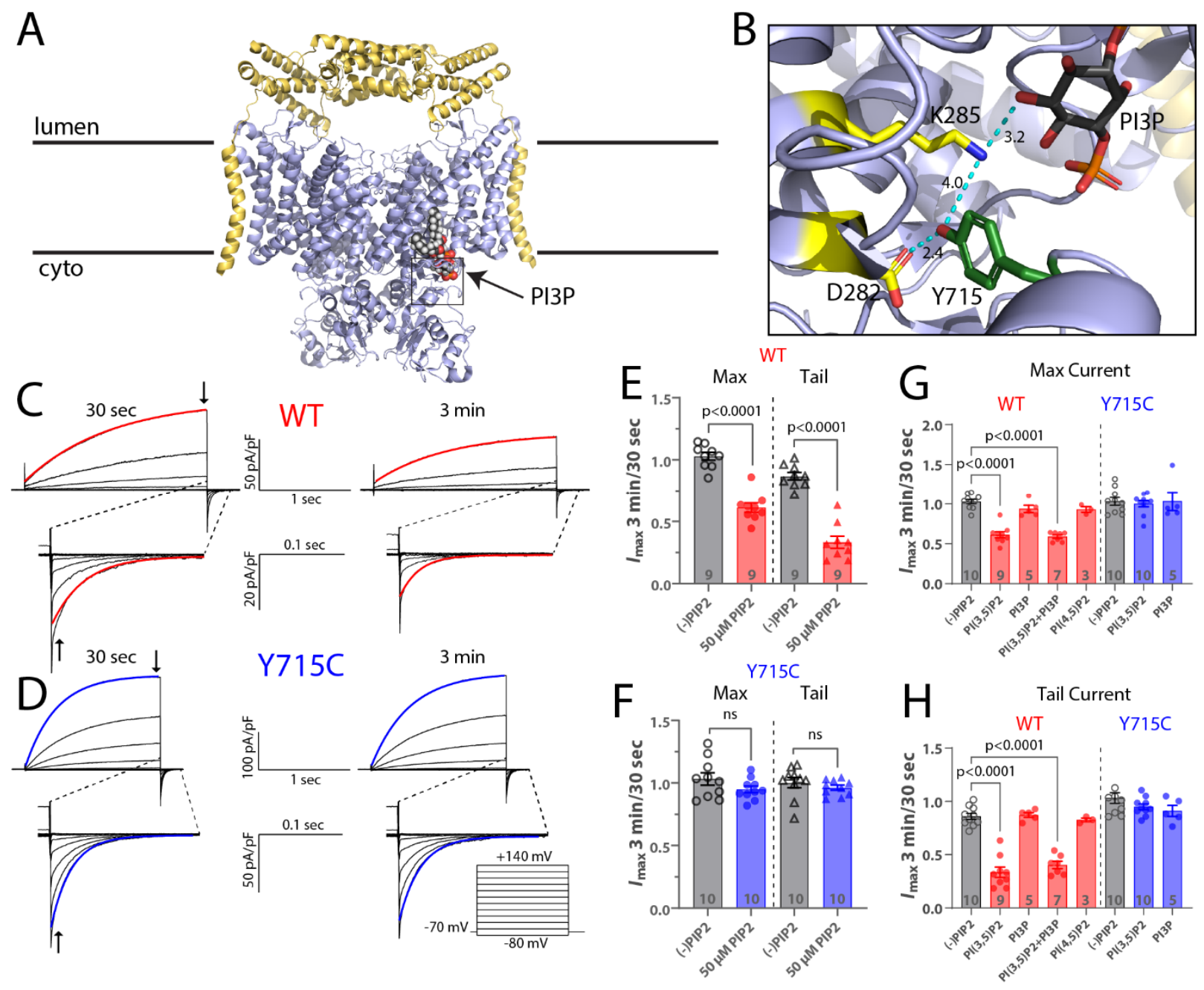

Figure 6. PI(3,5)P2 inhibits ClC-7 mediated currents. (A) Cryo-EM structure of human $\mathrm{ClC}-7$ (pale blue) and Ostm1 (yellow) (PDB 7JM7). PI3P is modeled bound at the interface between the transmembrane domain and the cytosolic CBS domains. (B) Enlarged view of the PI3P binding region boxed in (A). The structure reveals a hydrogen-bonding network involving the phospholipid head group and adjacent residues, including Y715. Measurement distances in angstroms are indicated. (C, D) Representative whole-cell current traces recorded from HEK-293 cells transfected with (C) wild-type or (D) Y715C mutant CIC-7. After allowing the pipette solution containing $50 \mu \mathrm{M} \mathrm{PI}(3,5) \mathrm{P} 2$ to diffuse into the cell for 3 minutes, maximum voltage-activated current magnitude and tail current magnitude on returning to holding potential were reduced in currents from wild-type but not mutant $\mathrm{ClC}$-7. Red or blue lines indicate a single exponential fit to obtain time constants. (E, F) The ratio of current magnitudes measured at 3 min and $30 \mathrm{sec}$ after cell break-in from (E) wild-type and (F) Y715C transfected cells as indicated by arrows in (C) and (D). Each point represents the ratio of two successive recordings from a single cell. Error bars represent standard error of the mean and $p$ values are calculated from a two-tailed unpaired Student's t-test. (G, H) Effect of 50 $\mu \mathrm{M}$ phospholipids on the change in $(\mathbf{G})$ maximum current and $(\mathbf{H})$ tail current after a $+140 \mathrm{mV}$ pulse as measured by the ratio of the indicated values 3 minutes and 30 seconds after cell break-in. Error bars represent standard error of the mean and adjusted $p$ values are calculated by Tukey's Method.

\section{The pathogenic mutation Y715C abolishes PI(3,5)P2 inhibition of ClC-7}

Recently, a novel disease-causing mutation in ClC-7, Y715C, was reported in two patients (Nicoli et al., 2019). At the lysosomal level, this variant is characterized by enlarged vacuoles and hyperacidified lysosomes, a phenotype remarkably similar to that observed in apilimod-treated cells. Consistent with 
these features, currents measured from cells expressing ClC-7-Y715C were larger than those expressing wild-type $\mathrm{ClC}-7$, suggesting that enhanced chloride/proton exchange could allow more $\mathrm{Cl}^{-}$to enter the lysosome and facilitate excess acidification, thereby contributing to the observed lower pH levels (Nicoli et al., 2019). Examination of the human CIC-7 structure reveals that the side chain of Tyrosine 715, located on the cytoplasmic domain, is within $10 \AA$ of the inositol ring of the bound PIP, and that it is involved in a hydrogen-bonding network with ring hydroxyls and several TM-domain residues (Figure $6 B)$.

We reasoned that this network might play an important role in coupling the binding of $\mathrm{PI}(3,5) \mathrm{P} 2$ to changes in transport currents. If so, the $\mathrm{Y} 715 \mathrm{C}$ mutation might perturb this interaction and limit the inhibitory effect of $\mathrm{PI}(3,5) \mathrm{P} 2$, resulting in the more active phenotype we previously described (Nicoli et al., 2019). To test this possibility, we repeated the PIP2 patch-clamp electrophysiology experiments described above after introducing the Y715C mutation. For currents from CIC-7PM Y715C, the inhibitory effect of PI(3,5)P2 we observed for WT transporters is no longer detectable; the phosphoinositide had essentially no effect on the mutant CIC-7 currents under these conditions (Fig. 6 D, F, Fig 6 Supplement 1D). This result suggests that the $\mathrm{Y} 715 \mathrm{C}$ mutation either weakens the binding of $\mathrm{PI}(3,5) \mathrm{P} 2$ to $\mathrm{ClC}-7$ or disrupts the coupling between lipid binding and the transporter gating. As with wild-type ClC-7, PI3P also had no detectable effect on $\mathrm{Y715C}$ mutant currents (Fig 6G,H, Fig 6 Supplement $1 \mathrm{C}$, D). Even increasing the pipette concentration of $\mathrm{PI}(3,5) \mathrm{P} 2$ to $100 \mu \mathrm{M}$ did not affect Y715C currents (not shown). These results demonstrate a mechanistic connection between the lysosomal effects observed under PIKfyve inhibition and those observed in patients carrying the $\mathrm{Y} 715 \mathrm{C}$ mutation: in the former case, a decrease in available $\mathrm{PI}(3,5) \mathrm{P} 2$ relieves a tonic inhibition of $\mathrm{CIC}-7$ by the lipid, and in the latter, $\mathrm{PI}(3,5) \mathrm{P} 2$ may be present but it is unable to elicit its usual inhibition of transport. 

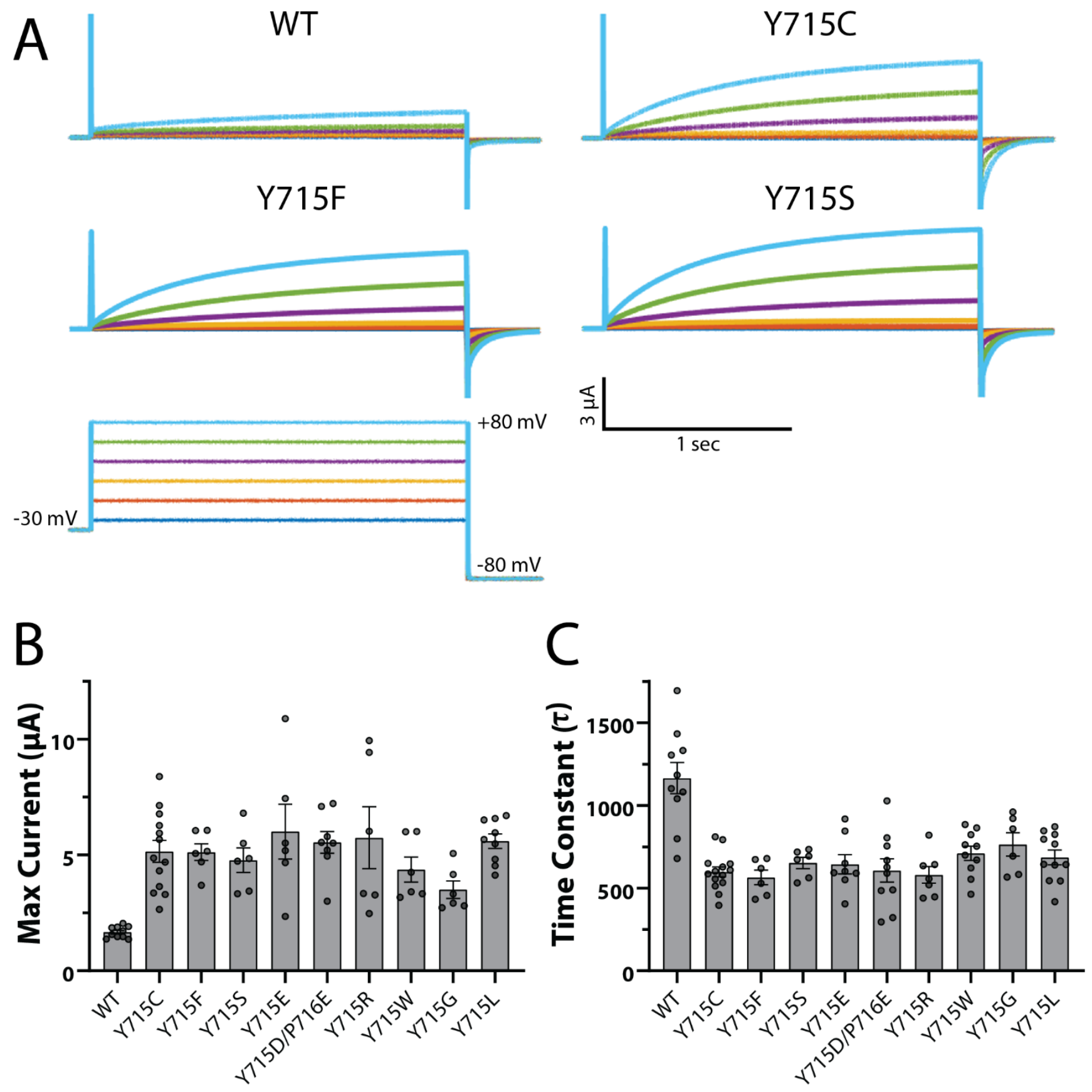

Figure 7: Y715 is required for proper CIC-7 function. Y715x mutations were introduced to the plasma-membrane targeted CIC-7 and currents measured in Xenopus oocytes under Two-Electrode Voltage Clamp. (A) Representative voltage families recorded from oocytes injected with the indicated construct. (B) Maximum current measured from ClC-7 expressing oocytes at the end of a $2 \mathrm{~s}+80 \mathrm{mV}$ voltage pulse. (C) Activation time constant at $+80 \mathrm{mV}$ obtained from a single exponential fit to the current trace. Each dot represents a value measured from an individual oocyte; 2-5 independent batches of oocytes were recorded for each mutant.

Close examination of the human $\mathrm{ClC}-7$ structure reveals that $\mathrm{Y} 715$ is engaged in a network of $\mathrm{H}$ bonds with residues on the protein's transmembrane domain that extends to the 5 ' hydroxyl on the PIP inositol ring (Fig 6B). We tested the importance of the hydroxyl group and phenyl ring on this tyrosine by 
mutating it to a phenylalanine or to a serine, respectively and measured transporter currents using the CIC-7PM expressed in Xenopus oocytes with two-electrode voltage clamp (Fig. 7). Removing either the hydroxyl group (Y715F) or the phenyl ring (Y715S) both increased maximal current at $+80 \mathrm{mV}$ and sped current activation. For each mutation we examined, the transporter current had characteristics nearly identical to that of the original $\mathrm{Y} 715 \mathrm{C}$ mutant, suggesting that the WT tyrosine is essential at this position. Indeed, multiple other mutations at this position all resulted in currents very similar to that of Y715C, strongly supporting the importance of the WT tyrosine for proper transporter function (Figs 7, Fig 7 Supplement 1).

\section{DISCUSSION}

In this study, we demonstrated that acute pharmacological inhibition of PIKfyve and consequent depletion of $\mathrm{PI}(3,5) \mathrm{P} 2$, induces a lysosomal hyperacidification of 0.3 to $0.5 \mathrm{pH}$ units, and that the effect is largely due to contributions from $\mathrm{ClC}-7$. Our mechanistic experiments revealing direct inhibition of CIC-7 by $\mathrm{PI}(3,5) \mathrm{P} 2$ suggest that tonic $\mathrm{PI}(3,5) \mathrm{P} 2$ inhibition of the $\mathrm{CIC}-7$ is relieved by the lipid depletion caused by apilimod inhibition of PIKfyve. We observed hyperacidification in multiple cell types, including U20S osteosarcoma cells, human neonatal fibroblasts and SUDHL B-cell non-Hodgkin lymphoma cells. Our results clearly establish that PIKfyve inhibition can affect lysosomal pH. There are several possible reasons why previous efforts to identify such changes were unsuccessful. First, it is essential to use a dye with sensitivity at the low end of the lysosomal pH range. Ho et al (Ho et al., 2015) used FITC as their pH sensing dye; with a pKa around 6.0 (Fig. 1 Supplement 3) the dye is minimally sensitive to pH below about 4.5 so changes in the range we observe would be difficult to detect. Recently, Sharma et al. measured lysosomal pH with OG and concluded that apilimod or a new PIKfyve inhibitor named WX8 don't affect lysosomal pH of RAW or U2OS cells (Sharma et al., 2019). However, their experiments yield untreated lysosomal $\mathrm{pH}$ values of $5.25 \pm 0.05$ units in both cell lines; this is above the range we observe and may indicate a problem with calibration or image processing. Nonetheless, that group did observe a (significant) hyperacidification of $\sim 0.2 \mathrm{pH}$ units with one inhibitor (WX8), consistent with our findings. Additional variability may result from cell-type differences in acidification control or in the role of PIKfyve (Sbrissa and Shisheva, 2005).

Apilimod-induced vacuoles originate from endolysosomes and lysosomes through alterations in the tubulation/fission processes that constantly reform these organelles (Bissig et al., 2017; Choy et al., 2018; Sharma et al., 2019). Although the molecular mechanism for such alteration remains unknown, Compton et al. showed (and our results replicated) that the proton pump V-ATPase must be active to 
produce vacuoles (Compton et al., 2016), though it has been suggested that these effects could result from off-target effects of BafA1 (Mauvezin et al., 2015). Our results using $\mathrm{pH}$-modifying agents early in vacuole development clearly demonstrate that the hyperacidic luminal $\mathrm{pH}$ resulting from apilimod treatment is neither responsible for initiation of vacuole formation, nor for the increase in vacuole size, as alkalinizing lysosomes to $\mathrm{pH}$ values above about $\mathrm{pH} 5.5$ had no impact on these effects. In further support of a separation of these processes, lysosomal tubulation and fission events (size decrease) precede the return to normal $\mathrm{pH}$. However lysosomal size and $\mathrm{pH}$ are not completely decoupled, as indicated by the observation that $\mathrm{ClC}-7$ knockout dampens both apilimod-induced size and pH changes, as well as by the effects of the disease-causing mutation CIC-7 Y715C (Nicoli et al., 2019). This shift could reflect alteration of lysosomal membrane electrical potential or changes of luminal ion concentrations, in turn affecting the trafficking, and/or inducing osmotic stress (Di Zanni et al., 2021; Freeman et al., 2020; Sartelet et al., 2014).

Our electrophysiology experiments reveal a direct and specific inhibitory effect of $\mathrm{PI}(3,5) \mathrm{P} 2$ on CIC-7 activity. Based on the changes caused by PI(3,5)P2 to the kinetics of slow CIC-7 voltage dependent activation and deactivation, we suggest that the lipid tunes this slow gating process rather than affecting the transport cycle itself. Notably, in the human CIC-7 structure the bound lipid is modeled as a PI3P, a lipid which did not perceptibly inhibit CIC-7 transport in our experiments and did not compete with $\mathrm{PI}(3,5) \mathrm{P} 2$ at concentrations we could achieve. Further work will be necessary to determine the role, if any, of PI3P in ClC-7 function. The headgroup of the bound lipid in the structure participates in a hydrogen bonding network that encompasses residues both on the TM domain and the cytoplasmic domain of the protein, and which includes the $Y 715$ residue that is mutated in the gain-of-function patient. Our observations here that the $\mathrm{Y} 715 \mathrm{C}$ mutation abolishes $\mathrm{PI}(3,5) \mathrm{P} 2$ inhibition, and that the Y715 position is exquisitely sensitive to mutation suggests a model wherein $\mathrm{ClC}-7$ voltage dependent gating comprises substantial conformational changes involving this interface, changes which we infer are modulated by the presence and identity of the bound lipid. Perhaps upon binding PI(3,5)P2 the 5phosphate revises the $\mathrm{H}$-bond network and induces a gating-controlling conformational change to inhibit the transporter. This model is consistent with a large body of evidence for a role of the cytoplasmic domain in slow gating of $\mathrm{ClC}-7$, including a report that the $\mathrm{ClC}-7 \mathrm{C}$-terminal domain is necessary for voltage-dependent gating that corroborates the importance of this interface (Di Zanni et al., 2021; Ludwig et al., 2013; Sartelet et al., 2014). 
The work we present here advances our understanding of the role of $\mathrm{ClC}-7$ in lysosomal acidification. Previous work has suggested that ClC-7 forms part of the lysosomal counterion pathway based on increases in lysosomal pH in cells with ClC-7 knocked down using siRNA (Graves et al., 2008) as well as the effect of $\mathrm{Cl}^{-}$removal from the bathing media in isolated lysosomes (Ohkuma et al., 1982). However, cells from multiple tissues in CIC-7 KO mice show normal lysosomal pH (Kasper et al., 2005; Wartosch et al., 2009), a conclusion reinforced by the retention of normal pH in the untreated ClC-7 KO cells we examined here. Our results suggest a possible explanation for this discrepancy: we hypothesize that $\mathrm{ClC}-7$ is largely inhibited by $\mathrm{PI}(3,5) \mathrm{P} 2$ in the normal cellular conditions, preventing it from strongly influencing baseline lysosomal $\mathrm{pH}$, but is activated by stimuli that decrease $\mathrm{PI}(3,5) \mathrm{P} 2$ concentrations, thereby providing additional $\mathrm{Cl}^{-}$as a counterion and facilitating further lysosomal acidification. This model is supported by the hyperacidification in patient-cell lysosomes carrying the $\mathrm{Y} 715 \mathrm{C}$ gain-offunction mutation (Nicoli et al., 2019). Such a mechanism could complement that observed in microglia, where trafficking of $\mathrm{ClC}-7$ to the lysosome upon activation by inflammatory stimuli caused acidification (Majumdar et al., 2011). The nature of the physiological stimuli for changes in $\mathrm{PI}(3,5) \mathrm{P} 2$ to regulate lysosomal pH remains to be determined, though the PIKfyve kinase has been shown to participate in multiple metabolic pathways (McCartney et al., 2014a).

Regulation of lysosomal $\mathrm{pH}$ is critical for normal cellular function; the complexity of this regulation is fertile ground for further study. Though the proton-pumping V-Type ATPase is clearly the metabolic driver of acidification, other factors, including the counterion pathway are also critical. In recent years a host of candidates for the counterion pathway have been identified, including ion channels like TRPML1 (Dong et al., 2010), TPC1 and TPC2 (Wang et al., 2012), and TMEM175 (Cang et al., 2015), and ion transporters like ClC-7 (Graves et al., 2008); indeed, several of these channels are also known to be modulated by PI(3,5)P2 (Fine et al., 2018; She et al., 2018; Wang et al., 2012). However, their physiological functions and contributions to different lysosomal processes are still emerging. Besides $\mathrm{pH}$ maintenance, lysosomal ions clearly contribute to multiple other processes, including triggering membrane fusion (Dong et al., 2010), driving the export of lysosomal degradation products (Sagné and Gasnier, 2008), maintaining osmotic balance (Freeman et al., 2020), and mediating lysosomal excitability (Cang et al., 2014), suggesting a need for coordinated control of multiple pathways. The work we present here uncovers a wealth of possibilities to study fundamental processes underlying lysosomal $\mathrm{pH}$ control and hints at complex interactions between multiple ion pathways to balance competing physiological requirements. Much work remains to understand the interplay of these factors and their pleiotropic effects. 


\section{MATERIALS AND METHODS}

\section{Chemicals}

Apilimod (Medchem Express); WX8 (gift from Juan S. Bonifacino lab) Oregon Green 488-dextran 10000mW (OG; ThermoFisher); Chloroquine, BafA1, Monensin and Nigericin (Sigma-Aldrich); Lysotracker blue DND-22 (Invitrogen); Magic red Cathepsin-B essay (ImmunoChemistry Technologies).

\section{Cell culture}

U2OS cells (HTB-96 ${ }^{\text {TM}}$ ) were purchased from ATCC and cultured in McCoy's 5A Modified Medium supplemented with $10 \% \mathrm{FBS}$ and $100 \mathrm{U} \cdot \mathrm{mL}^{-1}$ Penicillin-Streptomycin (all from gibco) at $37{ }^{\circ} \mathrm{C}$ in $5 \% \mathrm{CO}_{2}$ and $10 \%$ humidity. Cells were passed 2 times per week using TrypLE (gibco) and were used for experiments with a passage number lower than 40 . Human primary neonatal fibroblasts were also purchased from ATCC (PCS-201-010 ${ }^{\mathrm{TM}}$ ) and cultured in DMEM/F12 GlutaMAX medium supplemented with $10 \% \mathrm{FBS}$ and $100 \mathrm{U} \cdot \mathrm{mL}^{-1}$ Penicillin-Streptomycin at $37^{\circ} \mathrm{C}$ in $5 \% \mathrm{CO}_{2}$ and $10 \%$ humidity. Cells were passaged twice weekly; cells used in three independent apilimod treatment experiments were at passages 10, 12, and 16. All culture reagents were obtained from Gibco.

\section{OG Lysosome-loading procedure}

The day before imaging, cells were incubated overnight in a Nunc ${ }^{T M}$ Lab-Tek $^{T M}$ II 8 well chamber (ThermoFisher) in culture medium supplemented with $1 \mathrm{mg} / \mathrm{mL}$ OG for 15-16 h. OG was subsequently chased by washing at least $4 \mathrm{~h}$ before starting a protocol. For fibroblast experiments, cells were incubated with $2 \mathrm{mg} / \mathrm{mL} \mathrm{OG}$.

\section{Cell imaging}

Live cell imaging was performed at room temperature using a Nikon eclipse-Ti microscope with a 60X objective (Apo TIRF, NIKON) and a digital camera (ORCA-flash 4.0, Hamamatsu). Cells were imaged in an imaging media containing (in $\mathrm{mM}$ ): $150 \mathrm{NaCl}, 5 \mathrm{KCl}, 1 \mathrm{CaCl}_{2}, 1 \mathrm{MgCl}_{2}, 20 \mathrm{HEPES}, 10$ Glucose and 30 Sucrose, adjusted pH 7.2-7.4 with $\mathrm{NaOH}$. For $\mathrm{pH}$ measurements, images were acquired in epifluorescent mode using a $445 \mathrm{~nm}$ laser (OBIS, Coherent), a $488 \mathrm{~nm}$ laser (Sapphire, Coherent) and an >500 nm emission filter (ET500lp, Chroma). For each cell, separate images with each laser were obtained within 1 $\mathrm{s}$ of each other. For experiments using mCherry, we used the same excitation filterset to perform $\mathrm{pH}$ measurements but used a $535 \pm 35 \mathrm{~nm}$ emission filter (ET535/70m, Chroma) to eliminate crosstalk from the red channel. A SOLA light engine (lumencor) was used with an mCherry cube $(49008$, Chroma) to image for mCherry fluorescence. Image acquisition was performed using NIS-Element software (Nikon). 
For fibroblast lysosome $\mathrm{pH}$ measurements, epifluorescence images were acquired using a CoolLED pE4000 LED system. Images were acquired by excitation using the $435 \mathrm{~nm}$ LED or $490 \mathrm{~nm}$ LED with a $488 / 10 \mathrm{~nm}$ BrightLine ${ }^{\circledR}$ single-band bandpass excitation filter (Semrock) and the $435 \mathrm{~nm}$ LED.

\section{Image analysis}

Intensity and size of cell's OG positive objects were extracted using the software Slicer2D developed by Calvin Johnson and Huey Cheung from the Center for Information Technology from National Institutes of Health (Lau et al., 2013). Briefly, local background of $445 \mathrm{~nm}$ and $488 \mathrm{~nm}$ images were background subtracted and subsequently threshold using the Otsu method (Otsu, 1979) to select ROI corresponding to individual lysosomes. Each ROI is paired between the two images and a filter comparing paired ROI centroid and intensity is applied to discard single-image ROI or ROI containing low-intensity pixels value. Size, intensity and 488/445 ratio of each ROI are then calculated.

\section{pH calibration curve}

Just after imaging cells in protocol-based conditions, imaging media was washed out and replaced sequentially by a set of $\mathrm{pH}$ calibrated buffers supplemented with Monensin $\left(7.0 \mu \mathrm{g} . \mathrm{mL}^{-1}\right.$, dissolved in ethanol) and Nigericin (7.5 $\mu \mathrm{g} \cdot \mathrm{mL}^{-1}$, dissolved in ethanol) (0.29 \% ethanol in final solution) to equilibrate $\mathrm{pH}$ across cell and organelle membranes. $\mathrm{pH}$ calibration buffers composition: $\mathrm{pH}$ 7.0: Imaging media (IM) plus $40 \mathrm{mM}$ dibasic Sodium phosphate (NaP) and $10 \mathrm{mM}$ Tris-maleate (TM); pH 6.0: IM plus $25 \mathrm{mM} \mathrm{NaP}$ and $25 \mathrm{mM}$ TM; pH 5.0: IM plus $5 \mathrm{mM} \mathrm{NaP}$ and $45 \mathrm{mM} \mathrm{TM}$; pH 4.5: IM plus $30 \mathrm{mM} \mathrm{NaP}$ and $20 \mathrm{mM}$ citrate; $\mathrm{pH}$ 4.0: IM plus $26 \mathrm{mM} \mathrm{NaP}$ and $24 \mathrm{mM}$ citrate; $\mathrm{pH}$ 3.0: IM plus $20 \mathrm{mM} \mathrm{NaP}$ and $30 \mathrm{mM}$ citrate. For each buffer, lysosomal pH measures were obtained on 4-5 different cells. 488/445 nm ratios were then plotted in function of $\mathrm{pH}$ buffers and fitted by a 4-parameter sigmoidal function curve using Sigma plot v12 (Systat Software Inc.).

\section{CIC-7 KO CRISPR clone engineering}

To knock out ClC-7 at the genomic level in U2OS cells, two guide RNA plasmid constructs were created using lentiCRISPRv2 (Shalem et al., 2014; Sanjana et al., 2014). Target sites for deletion in EXON1 of CLCN7 were designed using a CRISPR design website (http://crispr.mit.edu/). The resulting target sequences were as follows: 5'-TGTCCTGGTCCGGCCGGGACCGG-3'; 5'-ACGCCGCTGCTGAACGGGGCTGG3'. Guide oligonucleotide sequences were: T-1F, 5'-CACCGTGTCCTGGTCCGGCCGGGAC-3'; T-1R, 5'AAACGTCCCGGCCGGACCAGGACAC-3'; T-2F, 5'-CACCGACGCCGCTGCTGAACGGGGC-3'; T-2R， 5'AAACGCCCCGTTCAGCAGCGGCGTC-3' . As described in Zhang lab protocol (Sanjana et al., 2014), 
each guide oligo pairs were then annealed, cloned and transformed to Stbl3 bacteria. The positive constructs were identified and confirmed by sequencing. U2OS cells transfection with engineered lentiCRISPRv2 were done using FuGENE 6 transfection reagent (Promega). Positive clones were selected using puromycin and subsequently isolated.

\section{U2OS cell genotyping and RT-qPCR amplification}

Genotyping of ClC-7 KO U2OS clone was performed using an Extract PCR-Kit (Bioline) with the following primers: GAGAACAAACACGGGGGCA; GCGTTCCCGAGTCCACC. Isolated total RNA from WT and KO U2OS cells (QIAGEN RNeasy Mini Kit) reverse transcribed into cDNA (BIORAD iScript ${ }^{\mathrm{TM}}$ cDNA Synthesis Kit) and primers (indicated as followed) were used to quantify mRNA expression levels by realtime PCR using iQ ${ }^{\mathrm{TM}}$ SYBR ${ }^{\circledR}$ Green Supermix (BioRad) and CFX96 Touch Real-time PCR Detection System (Bio-Rad). Primers used: GAPDH: 5'-GTCTCCTCTGACTTCAACAGCG-3'; 5'-ACCACCCTGTTGCTGTAGCCAA-3'. CIC-7: 5'-CCCACTCCAGCTCTTCTGTG-3'; 5'-ATAGGAGCCTGGTGGGTCATG-3'. Relative expression of CIC-7 mRNA was calculated by normalizing the expression values of WT and KO to those of GAPDH.

\section{mCherry, PIKfyve constructs and transfection procedure}

PIKfyve (sequence id. AY457063.1) and PIKfyve-KYA hyperactive mutant (E1620>K, N1630>Y, S2068>A) were cloned into PCMV-HA-N vector (Clontech) and gifted by Lois Weisman lab. mCherry (sequence id. AIL28759.1) was cloned into PCAGEN vector (addgene 11160) and gifted by Kenton Swartz lab. U2OS cells were transfected in DMEM media with Lipofectamine 2000 (ThermoFisher).

\section{PIKfyve Western Blot}

2X106 U2OS cells expressing or not PIKfyve or PIKfyve ${ }^{\mathrm{KYA}}$ were collected and sonicated to extract total-proteins. $100 \mu \mathrm{g}$ of total-proteins was then subjected to electrophoresis in $4-12 \%$ Bis-Tris Plus Gels (Novex ${ }^{\circledast}$, NW00125BOX) and transferred onto PVDF membrane using a Trans-Blot ${ }^{\circledR}$ Turbo $^{\mathrm{TM}}$ Transfer System (BIORAD). After blocking with $5 \%$ non-fat milk, PVDF membrane was incubated at $4{ }^{\circ} \mathrm{C}$ with 1:1000 ratio of PIKFYVE antibody (Invitrogen, PA5-13977) or 1:1000 ratio of $\alpha / \beta$-Tubulin antibody (Cell Signaling, 2148). Both primary antibodies were probed using goat anti-rabbit IgG secondary antibody conjugated with horseradish peroxidase (Jackson ImmunoResearch, West Grove, PA) and visualized by chemiluminescence (SuperSignal West Pico Chemiluminescent Substrate, ThermoFisher). Images were obtained using an Odyssey FC Imager (Dual-Mode Imaging System; 2-min integration time). 


\section{Electrophysiology}

HEK-293 (ATCC CRL-1573) cells were cultured in growth medium consisting of 90\% Dulbecco's modified Eagle's medium, 10\% fetal bovine serum, 100 units $\mathrm{ml}-1$ of penicillin-streptomycin, $4 \mathrm{mM} \mathrm{L-}$ glutamine, and $1 \mathrm{mM}$ sodium pyruvate (Gibco). Cells were cultured in 35-mm polystyrene dishes (Falcon) at $37{ }^{\circ} \mathrm{C}$ in the presence of $5 \% \mathrm{CO}_{2}$. Cells were transiently co-transfected with $700 \mathrm{ng}$ each of human wild-type ClC-7 or ClC-7-Y715C in a pIRES2-EGFP vector and human Ostm1 in a pCMV-6 vector. Both $\mathrm{ClC}-7$ constructs also included mutations to two lysosome-targeting motifs in the $\mathrm{N}$ terminus to cause trafficking to the plasma membrane (Leisle et al., 2011; Stauber and Jentsch, 2010), allowing for whole-cell measurements. Transfection was accomplished using Lipofectamine LTX with Plus reagent (Invitrogen). Transfection was performed approximately 40 hours prior to experiments.

On the day of patch clamp experiments, cells were released from culture dishes by brief exposure to $0.25 \%$ trypsin/EDTA, resuspended in supplemented DMEM, plated on glass coverslips, and allowed to recover for $1-2 \mathrm{~h}$ at $37^{\circ} \mathrm{C}$ in $5 \% \mathrm{CO}_{2}$. Whole-cell voltage-clamp current measurements were performed using an Axopatch 200B amplifier (Axon Instruments) and pClamp 11.1 software (Axon Instruments). Data were acquired at $10 \mathrm{kHz}$ and filtered at $1 \mathrm{kHz}$. Patch pipettes were pulled using a P97 laser puller (Sutter Instruments) from borosilicate glass capillaries (World Precision Instruments) and heat-polished using a MF-200 microforge (World Precision Instruments). Pipette resistance was 2-5 megohms in the extracellular solution. A reference electrode was placed in a separate chamber containing extracellular solution and connected to with a $2 \%$ agar bridge made from extracellular solution. Extracellular solution consisted of (in $\mathrm{mm}$ ) $\mathrm{NaCl} 130,5, \mathrm{MgCl}_{2}$ 1, $\mathrm{CaCl}_{2}$ 1, HEPES 20, and glucose 20, with the $\mathrm{pH}$ adjusted to 7.4 using $\mathrm{NaOH}$ and the osmolality adjusted to 310 mOsmol using sucrose. Pipette solution contained (in $\mathrm{mm}$ ) $\mathrm{CsCl} 110, \mathrm{NaCl} 10, \mathrm{MgCl}_{2}$ 2, EGTA 1, and HEPES 40; pH was adjusted to 7.2 with $\mathrm{CsOH}$, and osmolality was adjusted to $300 \mathrm{mOsmol}$ using sucrose. Chemicals were obtained from Sigma. Osmolality was measured using a Vapro 5600 vapor pressure osmometer (Wescor). For measurements in the presence of PIPs, PI(3,5)P2 diC8 (Echelon Biosciences P-3508), PI3P (Echelon Biosciences P-3008), or PI(4,5)P2 (Echelon Biosciences P-4508) was dissolved in water at a concentration of $2 \mathrm{mM}$ and stored at $-80^{\circ} \mathrm{C}$ in small aliquots; on the day of experiments, stock PIP2 was diluted into pipette solution to a final concentration of $50 \mu \mathrm{M}$ and kept on ice during the course of the experiment.

\section{Statistical analysis}

Data are reported as mean \pm Standard Error of the Mean (SEM) and were analyzed using Prism 8 (Graphpad). Each statistical test used is indicated in the legend of the corresponding figure. 


\section{ACKNOWLEDGMENTS}

We thank LS Weisman for providing us the PIKfyve ${ }^{\mathrm{KYA}}$ construct, K Swartz for providing the mCherry construct, and ML DePamphilis for the WX8 compound. $K$ Swartz and C Lingle provided critical readings of the manuscript and valuable discussions. This work was supported by the NINDS intramural program.

\section{COMPETING INTERESTS}

The authors declare no competing interests.

\section{AUTHOR CONTRIBUTIONS}

Conceptualization: XL, JH, JAM; Methodology: MW, XL, JH, JM; Investigation: XL, JH, MW, AB, KN; Writing-Original Draft: XL, JH, JAM; Writing-Review and Editing; XL, JH, VM, GF, JAM; Resources: JAM; Funding: JAM. Supervision: JAM 
bioRxiv preprint doi: https://doi.org/10.1101/2021.10.07.463477; this version posted October 7, 2021. The copyright holder for this preprint (which was not certified by peer review) is the author/funder. This article is a US Government work. It is not subject to copyright under 17 USC 105 and is also made available for use under a CCO license.

FIGURE 1 Supplement 1: Oregon Green 488-dextran loaded vesicles represent most of the late endocytic and lysosomal compartments. 3 channel colocalization images of a U2OS cell stained with Lysotracker blue, Magic-Red, and Oregon Green 488-dextran. Lysotracker blue and Magic Red stain cell's acidic compartments and Cathepsin-B positive compartments, respectively.
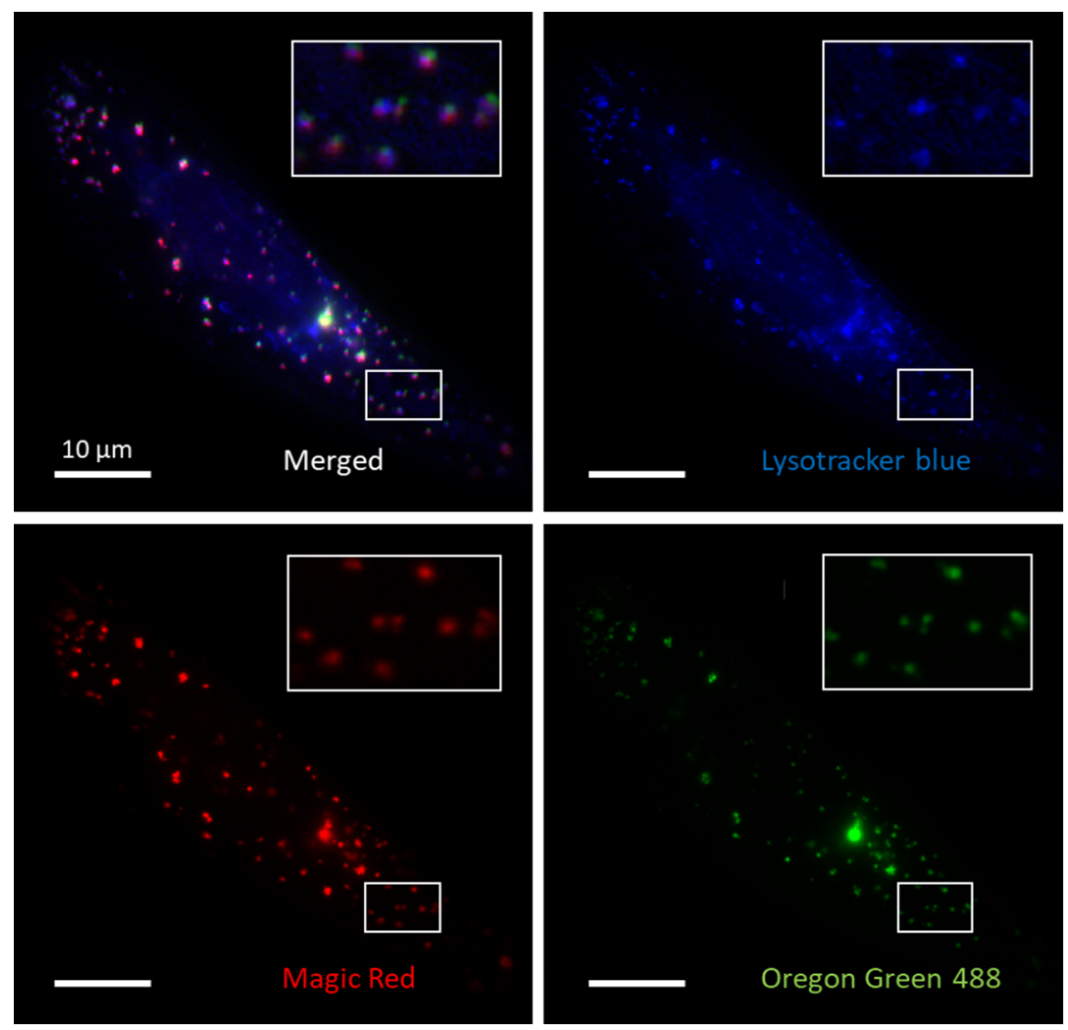
Figure 1 Supplement 2. PIKfyve inhibition causes hyperacidification in neonatal fibroblasts and SUDHL cells. Plots of the pH change (A) or ratio change (B) caused by $100 \mu \mathrm{M}(\mathrm{A})$ or $200 \mu \mathrm{M}$ (B) apilimod on either neonatal fibroblasts or SUDHL cells. Note that the 488/440 ratio is shown for SUDHL cells rather than $\mathrm{pH}$ because these cells were poorly adherent to glass coverslips and washed away on attempts to change solutions for $\mathrm{pH}$ calibration.

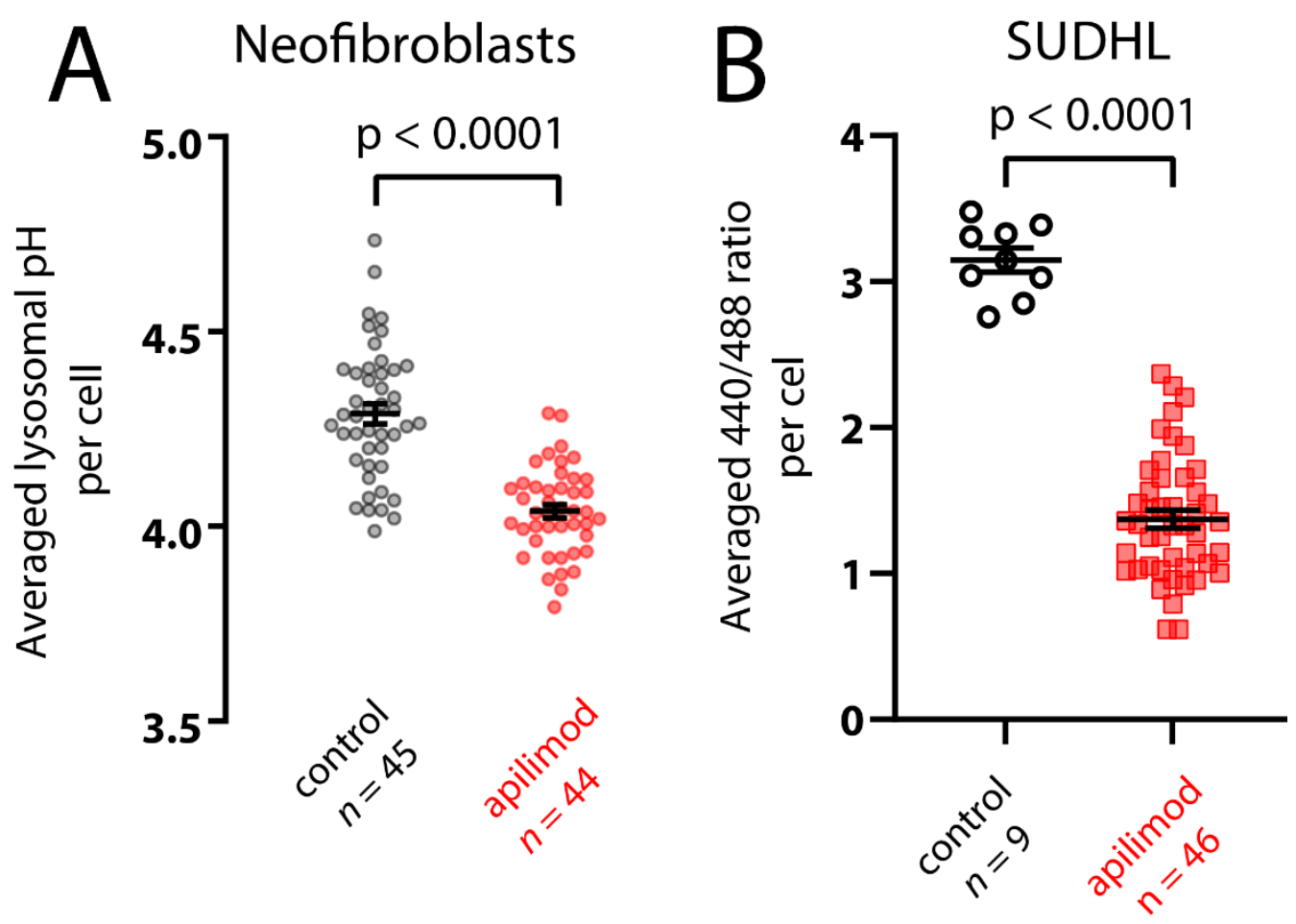


FIGURE 1 Supplement 3: pH calibration curve of RAW 264.7 lysosomes loaded with Fluorescein isothiocyanate-dextran 70000mW (FITC-dextran). Each dot represents the averaged lysosomal 488/445 ratio of one cell (4 cells per condition).

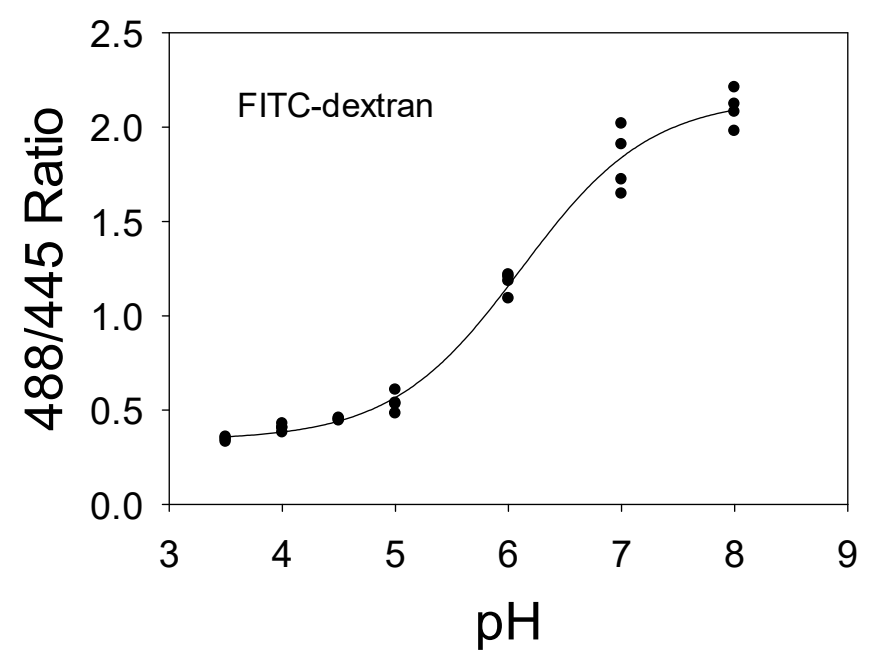

FIGURE 2 Supplement 1: Under PIKfyve inhibition, lysosomal pH does not correlate with lysosomal size. Representative plot of pH versus size of OG loaded lysosomes after 30min (left) or $1 \mathrm{~h}$ (right) apilimod treatment. We calculated the linear correlation to give a gross estimation of the relation between the two parameters (red dashed line). Populations of each graph represent all lysosomes from the experiment used in Fig. 2, C and D (11 cells, 632 lysosomes and 13 cells, 771 lysosomes for 30min and $1 \mathrm{~h}$ apilimod treatment, respectively).

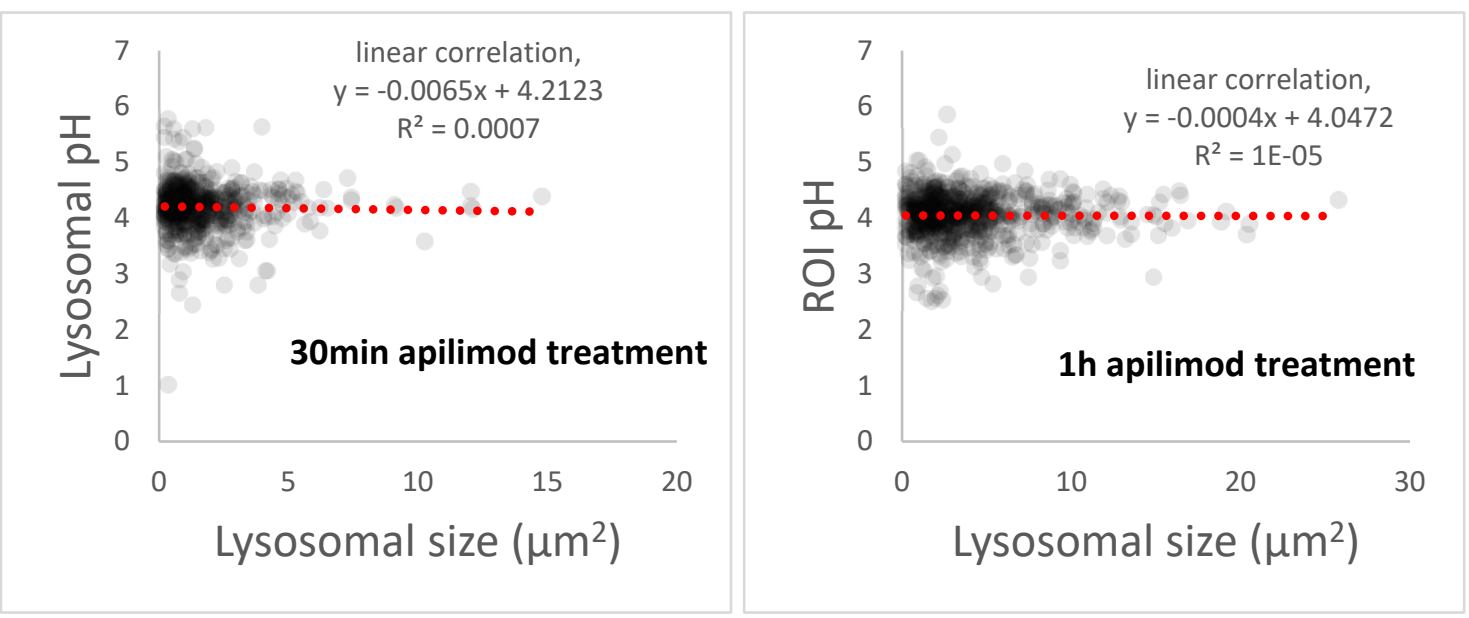


FIGURE 3 Supplement 1: $30 \mathrm{~min}$ treatment with chloroquine is sufficient to induce alkalization of endo/lysosomal compartments. Two representative experiments are shown. The level of alkalization after $30 \mathrm{~min}(\mathrm{red})$ is comparable to the one after $3 \mathrm{~h}$ chloroquine (blue) treatment. Two independent experiments are represented. "chloro." is for chloroquine.

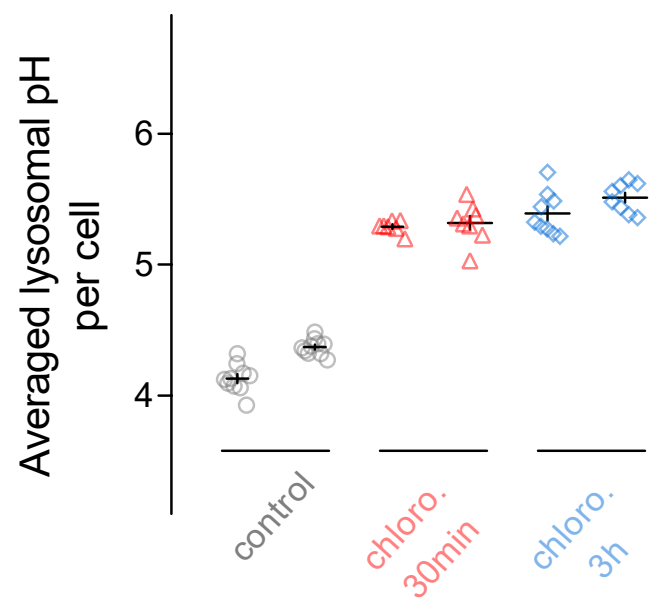

FIGURE 5 Supplement 1: Validation of the U2OS CIC-7 KO clone. (A) RT-qPCR amplification plot obtained from ClC-7 Wt (grey) and CIC-7 KO (red) U2OS cells whole RNA. GAPDH housekeeping RNA level (left-grey and left-red traces) is used as a control of RNA quantity. CICN7 RNA level corresponds to right-grey and right-red traces. $\Delta=3.5$ cycles, indicating a 90\% decrease in KO ClC-7 RNA level compared to Wt. (B) Agarose gel electrophoresis of qPCR product from ClC-7 Wt (grey) and ClC-7 KO (red) U2OS cells GAPDH or CLCN7 cDNA.

A

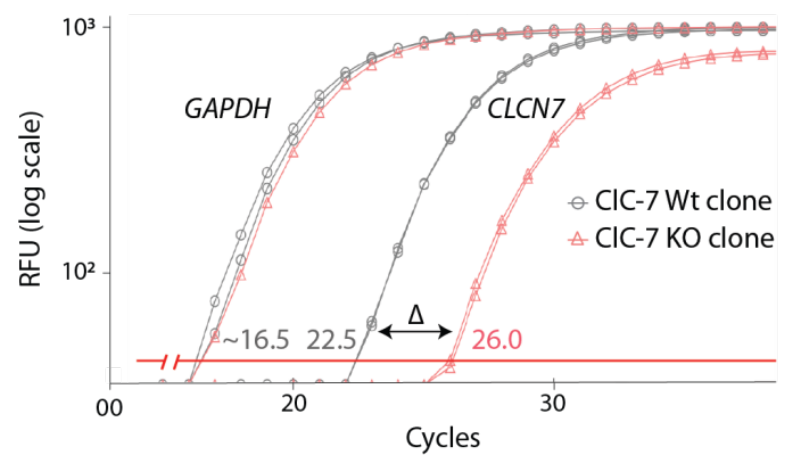

B

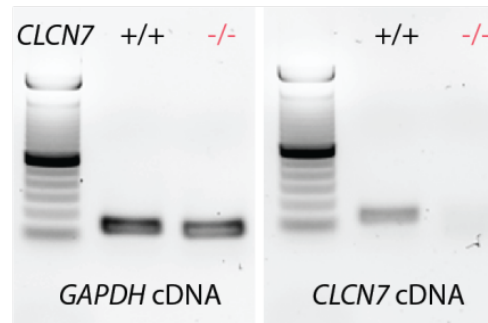


bioRxiv preprint doi: https://doi.org/10.1101/2021.10.07.463477; this version posted October 7, 2021. The copyright holder for this preprint (which was not certified by peer review) is the author/funder. This article is a US Government work. It is not subject to copyright under 17 USC 105 and is also made available for use under a CCO license. 
FIGURE 5 Supplement 2: CLCN7 deletion doesn't affect "lysosome-loading" with OG and doesn't alter pH measurements. Left: pH calibration curves of U2OS CIC-7 Wt (WT) or ClC-7 KO (KO) lysosomes loaded with OG in untreated (control) or 3h apilimod-treated conditions (apilimod). Each dot represents the averaged lysosomal 488/445 ratio of one cell (4 cells per condition). Right: Comparison of cumulative distribution of U2OS CIC-7 Wt (grey, control, 9 cells, 427 objects) versus CIC-7 KO (blue, 10 cells, 548 objects) lysosomal pH population. Each symbol represents the proportion of lysosomes having a $\mathrm{pH}$ value below the $\mathrm{pH}$ value represented in the abscissa axis.
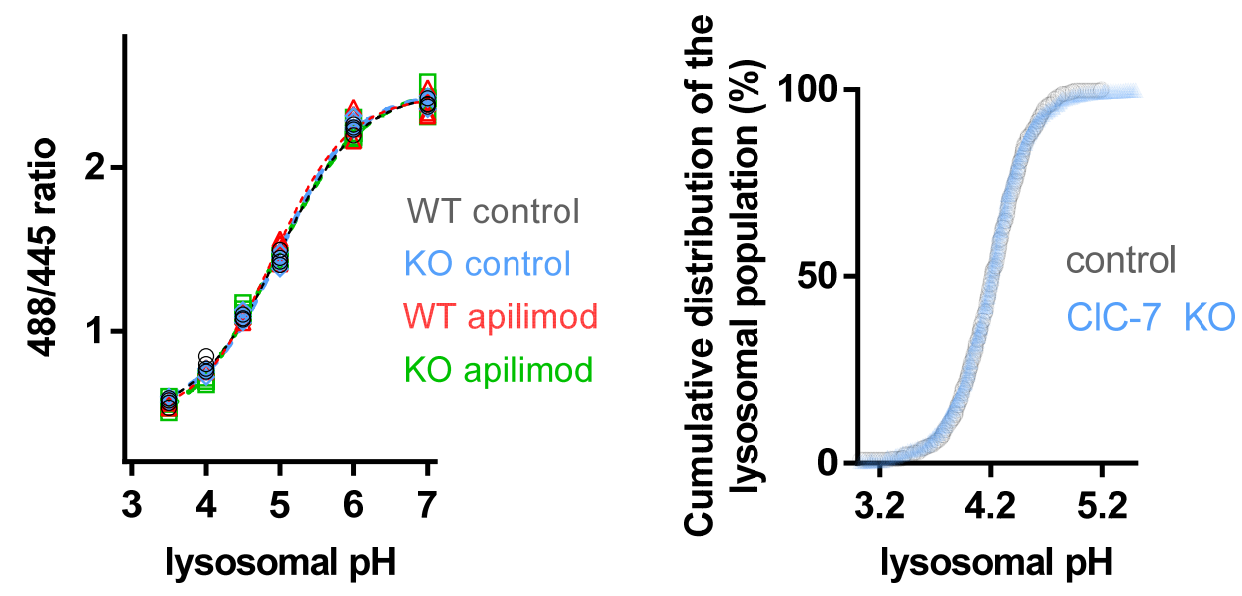
Figure 6 Supplement 1: Effect of phosphoinositides on CIC-7 currents in HEK cells. (A, B) Ratio of time constants obtained from single exponential fits of patch clamp current traces after a $+140 \mathrm{mV}$ pulse (activation) and subsequent return to $-70 \mathrm{mV}$ holding potential (deactivation). Currents were measured from HEK-293 cells transiently expressing (A) wild type CIC-7 or (B) CIC-7-Y715C. (C, D) Ratio of time constants after exposure to $50 \mu \mathrm{M}$ of the indicated phosphoinositide. (E) Dose dependence of CIC-7 inhibition by $\mathrm{PI}(3,5) \mathrm{P} 2$. Each point represents the average value from $n \geq 6$ HEK-293 cells transiently transfected with wild-type CIC-7.
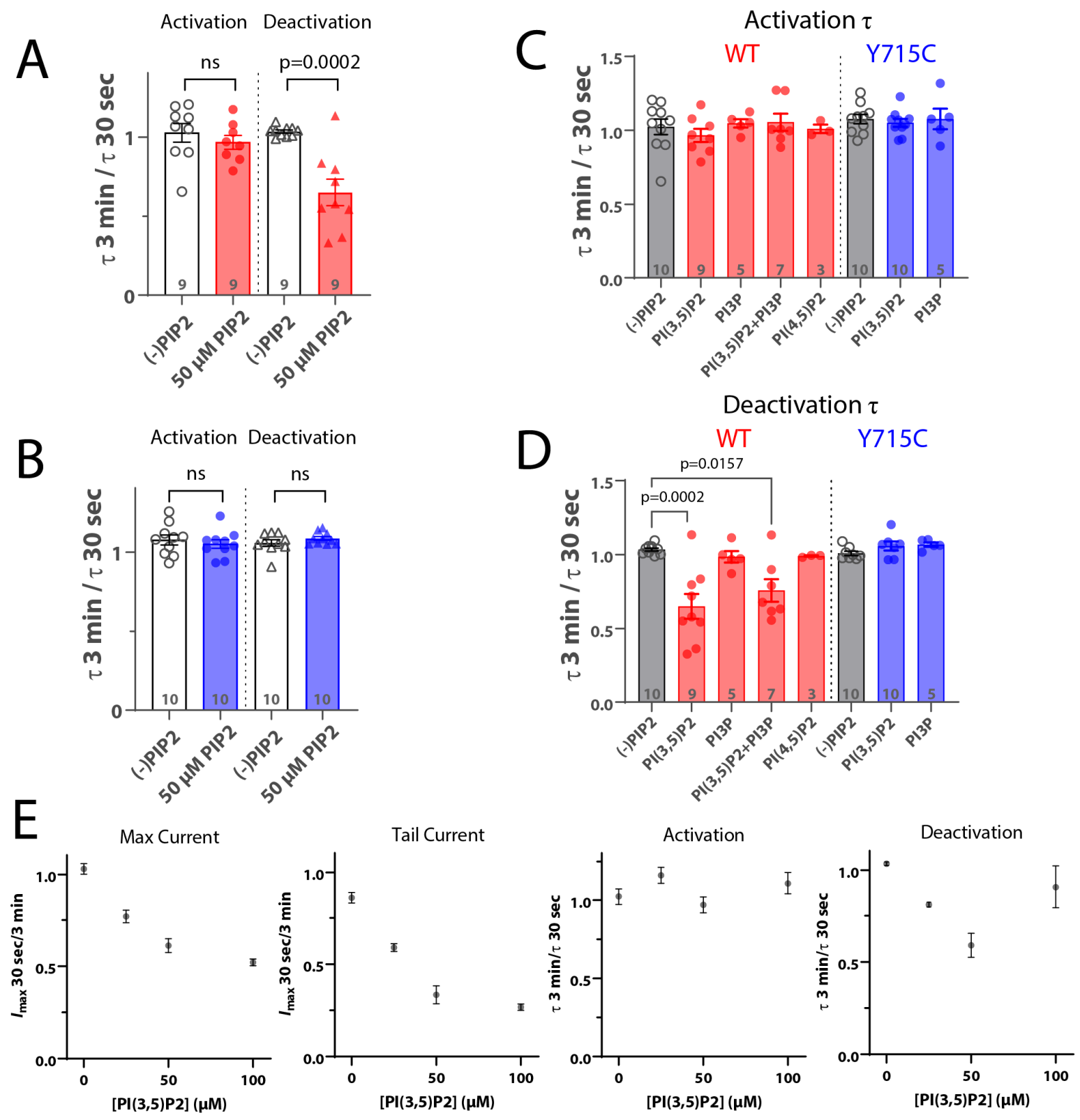
Figure 7 Supplement 1: The side chain of Y715 is essential for preserving wild-type function of

CIC-7. Representative voltage families recorded from oocytes injected with the indicated construct. All tested mutations resulted in a similar gain-of-function phenotype.

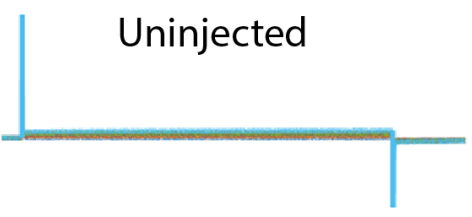

Y715F

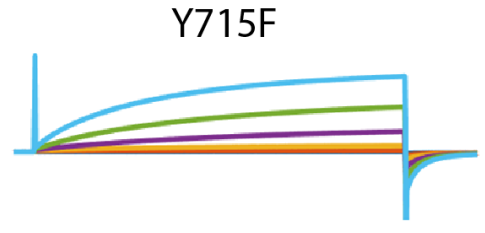

Y715D/Y715E
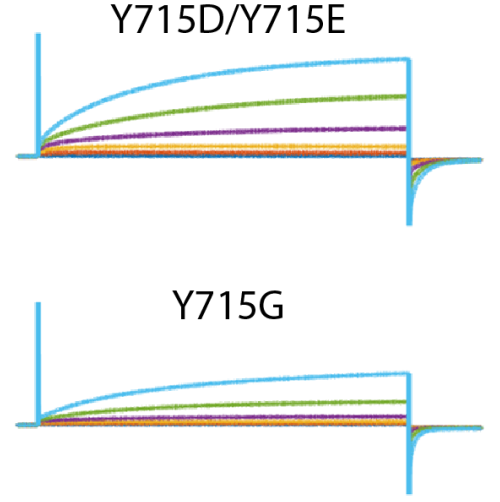

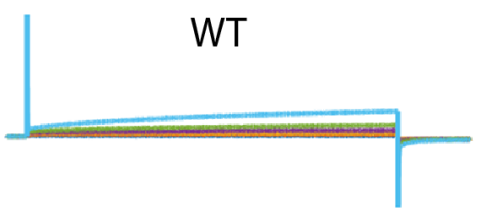

Y715S

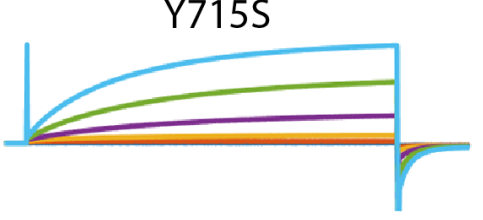

Y715R

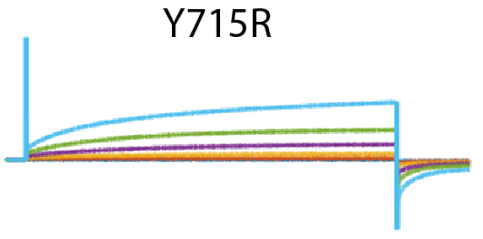

Y715L

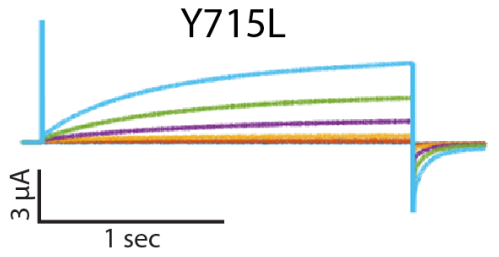

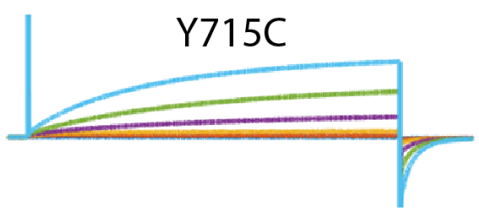

Y715E

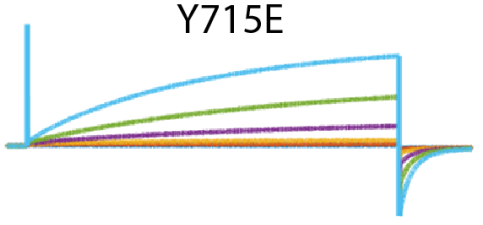

Y715W

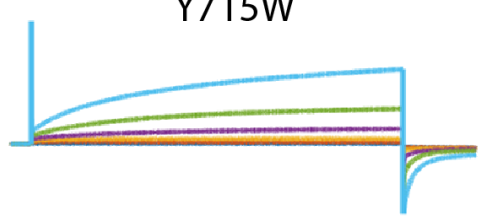

$-30 \mathrm{mV}$

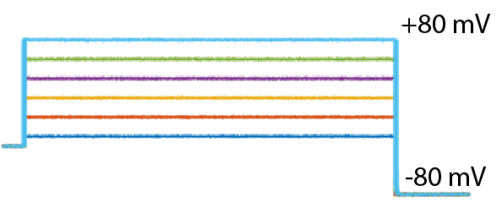




\section{LEGENDS FOR SOURCE DATA FILES}

Figure 4B Source Data Raw Blot Tubulin: merged images combining a light image of the blot itself and the chemiluminescent image of the blot probed with antitubulin antibody.

Figure 4B Source Data Raw Blot Tubulin: merged images of the same blot as above, but probed with an anti-PIKfyve antibody

Figure 4B-Source Data Labeled Gel: the above gel images with labels

Figure 5A Source Data Raw Gel: Agarose gel of PCR product amplified from CLCN7 deletion site of WT and CLCN7 KO U2OS cells

Figure 5A source data labeled gel: same gel as above with labels

Figure 5 supplement 1 Source Data Raw Gel: Agarose gel of RT-PCR products of GAPDH (positive control), CIC-7 WT, and CIC-7 KO U2OS cells.

Figure 5 Supplement 1 Source Data labeled Gel: Labeled gel of above 


\section{REFERENCES}

Bissig, C., Hurbain, I., Raposo, G., and van Niel, G. (2017). PIKfyve activity regulates reformation of terminal storage lysosomes from endolysosomes. Traffic 18, 747-757. 10.1111/tra.12525.

Bykova, E.A., Zhang, X.D., Chen, T.Y., and Zheng, J. (2006). Large movement in the C terminus of CLC-0 chloride channel during slow gating. Nat Struct Mol Biol 13, 1115-1119.

Cang, C., Aranda, K., Seo, Y.J., Gasnier, B., and Ren, D. (2015). TMEM175 Is an Organelle K(+) Channel Regulating Lysosomal Function. Cell 162, 1101-1112. 10.1016/j.cell.2015.08.002.

Cang, C., Bekele, B., and Ren, D. (2014). The voltage-gated sodium channel TPC1 confers endolysosomal excitability. Nature Chemical Biology 10, 463-469. 10.1038/nchembio.1522.

Carpaneto, A., Boccaccio, A., Lagostena, L., Di Zanni, E., and Scholz-Starke, J. (2017). The signaling lipid phosphatidylinositol-3,5-bisphosphate targets plant CLC-a anion/H+ exchange activity. EMBO reports 18 , 1100-1107. 10.15252/embr.201643814.

Chow, C.Y., Zhang, Y., Dowling, J.J., Jin, N., Adamska, M., Shiga, K., Szigeti, K., Shy, M.E., Li, J., Zhang, X., et al. (2007). Mutation of FIG4 causes neurodegeneration in the pale tremor mouse and patients with CMT4J. Nature 448, 68-72. 10.1038/nature05876.

Choy, C.H., Saffi, G., Gray, M.A., Wallace, C., Dayam, R.M., Ou, Z.-Y.A., Lenk, G., Puertollano, R., Watkins, S.C., and Botelho, R.J. (2018). Lysosome enlargement during inhibition of the lipid kinase PIKfyve proceeds through lysosome coalescence. Journal of Cell Science 131, jcs213587. 10.1242/jcs.213587.

Collins, D., Marcus, and Gordon, E., Sharona (2013). Short-Chain Phosphoinositide Partitioning into Plasma Membrane Models. Biophysical Journal 105, 2485-2494. 10.1016/j.bpj.2013.09.035.

Compton, L.M., Ikonomov, O.C., Sbrissa, D., Garg, P., and Shisheva, A. (2016). Active vacuolar H+ ATPase and functional cycle of Rab5 are required for the vacuolation defect triggered by Ptdlns $(3,5) \mathrm{P} 2$ loss under PIKfyve or Vps34 deficiency. American Journal of Physiology-Cell Physiology 311, C366-C377. 10.1152/ajpcell.00104.2016.

Di Zanni, E., Palagano, E., Lagostena, L., Strina, D., Rehman, A., Abinun, M., De Somer, L., Martire, B., Brown, J., Kariminejad, A., et al. (2021). Pathobiologic Mechanisms of Neurodegeneration in Osteopetrosis Derived From Structural and Functional Analysis of 14 ClC-7 Mutants. J Bone Miner Res 36, 531-545. 10.1002/jbmr.4200.

Dong, X.-P., Shen, D., Wang, X., Dawson, T., Li, X., Zhang, Q., Cheng, X., Zhang, Y., Weisman, L.S., Delling, M., and $\mathrm{Xu}, \mathrm{H}$. (2010). PI(3,5)P2 controls membrane trafficking by direct activation of mucolipin Ca2+ release channels in the endolysosome. Nature Communications 1, 1-11. 10.1038/ncomms1037.

Fine, M., Schmiege, P., and Li, X. (2018). Structural basis for PtdlnsP2-mediated human TRPML1 regulation. Nature Communications 9. 10.1038/s41467-018-06493-7.

Freeman, S.A., Uderhardt, S., Saric, A., Collins, R.F., Buckley, C.M., Mylvaganam, S., Boroumand, P., Plumb, J., Germain, R.N., Ren, D., and Grinstein, S. (2020). Lipid-gated monovalent ion fluxes regulate endocytic traffic and support immune surveillance. Science 367, 301-305. 10.1126/science.aaw9544.

Gayle, S., Landrette, S., Beeharry, N., Conrad, C., Hernandez, M., Beckett, P., Ferguson, S.M., Mandelkern, T., Zheng, M., Xu, T., et al. (2017). Identification of apilimod as a first-in-class PIKfyve kinase inhibitor for treatment of B-cell non-Hodgkin lymphoma. Blood 129, 1768-1778. 10.1182/blood-201609-736892. 
Graves, A.R., Curran, P.K., Smith, C.L., and Mindell, J.A. (2008). The $\mathrm{Cl}(-) / \mathrm{H}(+)$ antiporter ClC-7 is the primary chloride permeation pathway in lysosomes. Nature 453, 788-792. 10.1038/nature06907.

Hasegawa, J., Strunk, B.S., and Weisman, L.S. (2017). PI5P and PI(3,5)P<sub $>2</$ sub $>$ : Minor, but Essential Phosphoinositides. Cell Structure and Function 42, 49-60. 10.1247/csf.17003.

Ho, C.Y., Choy, C.H., Wattson, C.A., Johnson, D.E., and Botelho, R.J. (2015). The Fab1/PIKfyve Phosphoinositide Phosphate Kinase Is Not Necessary to Maintain the pH of Lysosomes and of the Yeast Vacuole. Journal of Biological Chemistry 290, 9919-9928. 10.1074/jbc.m114.613984.

Ikonomov, O.C., Sbrissa, D., and Shisheva, A. (2001). Mammalian Cell Morphology and Endocytic Membrane Homeostasis Require Enzymatically Active Phosphoinositide 5-Kinase PIKfyve. Journal of Biological Chemistry 276, 26141-26147. 10.1074/jbc.m101722200.

Ishida, Y., Nayak, S., Mindell, J.A., and Grabe, M. (2013). A model of lysosomal pH regulation. J Gen Physiol 141, 705-720. 10.1085/jgp.201210930.

Jentsch, T.J., and Pusch, M. (2018). CLC Chloride Channels and Transporters: Structure, Function, Physiology, and Disease. Physiological Reviews 98, 1493-1590. 10.1152/physrev.00047.2017.

Jin, N., Lang, M.J., and Weisman, L.S. (2016). Phosphatidylinositol 3,5-bisphosphate: regulation of cellular events in space and time. Biochem Soc Trans 44, 177-184. 10.1042/BST20150174.

Kasper, D., Planells-Cases, R., Fuhrmann, J.C., Scheel, O., Zeitz, O., Ruether, K., Schmitt, A., Poet, M., Steinfeld, R., Schweizer, M., et al. (2005). Loss of the chloride channel CIC-7 leads to lysosomal storage disease and neurodegeneration. Embo J 24, 1079-1091.

Kornak, U., Kasper, D., Bosl, M.R., Kaiser, E., Schweizer, M., Schulz, A., Friedrich, W., Delling, G., and Jentsch, T.J. (2001). Loss of the CIC-7 chloride channel leads to osteopetrosis in mice and man. Cell 104, 205-215.

Lange, P.F., Wartosch, L., Jentsch, T.J., and Fuhrmann, J.C. (2006). ClC-7 requires Ostm1 as a betasubunit to support bone resorption and lysosomal function. Nature 440, 220-223.

Lau, W.W., Johnson, C.A., Lioi, S., and Mindell, J.A. (2013). Three-Dimensional Spot Detection in Ratiometric Fluorescence Imaging For Measurement of Subcellular Organelles. ACM Conf Bioinform Comput Biol Biomed Inform (2013) 2013, 722. 10.1145/2506583.2512387.

Leisle, L., Ludwig, C.F., Wagner, F.A., Jentsch, T.J., and Stauber, T. (2011). CIC-7 is a slowly voltage-gated $2 \mathrm{Cl}(-) / 1 \mathrm{H}(+)$-exchanger and requires Ostm1 for transport activity. EMBO J 30, 2140-2152. emboj2011137.

Levine, B., Mizushima, N., and Virgin, H.W. (2011). Autophagy in immunity and inflammation. Nature 469, 323-335. 10.1038/nature09782.

Ludwig, C.F., Ullrich, F., Leisle, L., Stauber, T., and Jentsch, T.J. (2013). Common Gating of Both CLC Transporter Subunits Underlies Voltage-dependent Activation of the $2 \mathrm{Cl}-/ 1 \mathrm{H}+$ Exchanger $\mathrm{ClC}-7 / \mathrm{Ostm} 1$. Journal of Biological Chemistry 288, 28611-28619. 10.1074/jbc.m113.509364.

Majumdar, A., Capetillo-Zarate, E., Cruz, D., Gouras, G.K., and Maxfield, F.R. (2011). Degradation of Alzheimer's amyloid fibrils by microglia requires delivery of ClC-7 to lysosomes. Mol Biol Cell 22, 16641676. 10.1091/mbc.E10-09-0745.

Mauvezin, C., Nagy, P., Juhász, G., and Neufeld, T.P. (2015). Autophagosome-lysosome fusion is independent of V-ATPase-mediated acidification. Nature Communications 6, 7007. 10.1038/ncomms8007. 
Mccartney, A.J., Zhang, Y., and Weisman, L.S. (2014a). Phosphatidylinositol 3,5-bisphosphate: Low abundance, high significance. BioEssays 36, 52-64. 10.1002/bies.201300012.

McCartney, A.J., Zolov, S.N., Kauffman, E.J., Zhang, Y., Strunk, B.S., Weisman, L.S., and Sutton, M.A. (2014b). Activity-dependent PI(3,5)P2synthesis controls AMPA receptor trafficking during synaptic depression. Proceedings of the National Academy of Sciences 111, E4896-E4905.

10.1073/pnas.1411117111.

Mindell, J.A. (2012). Lysosomal Acidification Mechanisms. Annual Review of Physiology, Vol 74 74, 6986. 10.1146/annurev-physiol-012110-142317.

Müller, S., Dennemärker, J., and Reinheckel, T. (2012). Specific functions of lysosomal proteases in endocytic and autophagic pathways. Biochimica et Biophysica Acta (BBA) - Proteins and Proteomics 1824, 34-43. 10.1016/j.bbapap.2011.07.003.

Nicoli, E.-R., Weston, M.R., Hackbarth, M., Becerril, A., Larson, A., Zein, W.M., Baker, P.R., Burke, J.D., Dorward, H., Davids, M., et al. (2019). Lysosomal Storage and Albinism Due to Effects of a De Novo CLCN7 Variant on Lysosomal Acidification. The American Journal of Human Genetics 104, 1127-1138. 10.1016/j.ajhg.2019.04.008.

Ohkuma, S., Moriyama, Y., and Takano, T. (1982). Identification and characterization of a proton pump on lysosomes by fluorescein-isothiocyanate-dextran fluorescence. Proc Natl Acad Sci U S A 79, 27582762.

Saftig, P., and Klumperman, J. (2009). Lysosome biogenesis and lysosomal membrane proteins: trafficking meets function. Nature Reviews Molecular Cell Biology 10, 623-635. 10.1038/nrm2745.

Sagné, C., and Gasnier, B. (2008). Molecular physiology and pathophysiology of lysosomal membrane transporters. Journal of Inherited Metabolic Disease 31, 258-266. 10.1007/s10545-008-0879-9.

Sartelet, A., Stauber, T., Coppieters, W., Ludwig, C.F., Fasquelle, C., Druet, T., Zhang, Z., Ahariz, N., Cambisano, N., Jentsch, T.J., and Charlier, C. (2014). A missense mutation accelerating the gating of the lysosomal $\mathrm{Cl}$-/H+-exchanger $\mathrm{ClC}-7 / \mathrm{Ostm} 1$ causes osteopetrosis with gingival hamartomas in cattle. Dis Model Mech 7, 119-128. 10.1242/dmm.012500.

Sbrissa, D., and Shisheva, A. (2005). Acquisition of unprecedented phosphatidylinositol 3,5-bisphosphate rise in hyperosmotically stressed 3T3-L1 adipocytes, mediated by ArPIKfyve-PIKfyve pathway. J Biol Chem 280, 7883-7889. 10.1074/jbc.M412729200.

Schrecker, M., Korobenko, J., and Hite, R.K. (2020). Cryo-EM structure of the lysosomal chloride-proton exchanger CLC-7 in complex with OSTM1. eLife 9. 10.7554/elife.59555.

Settembre, C., Fraldi, A., Medina, D.L., and Ballabio, A. (2013). Signals from the lysosome: a control centre for cellular clearance and energy metabolism. Nat Rev Mol Cell Biol 14, 283-296.

$10.1038 / \mathrm{nrm} 3565$.

Shalem, O., Sanjana, N.E., Hartenian, E., Shi, X., Scott, D.A., Mikkelson, T., Heckl, D., Ebert, B.L., Root, D.E., Doench, J.G., and Zhang, F. (2014). Genome-scale CRISPR-Cas9 knockout screening in human cells. Science 343, 84-87. 10.1126/science.1247005.

Sharma, G., Guardia, C.M., Roy, A., Vassilev, A., Saric, A., Griner, L.N., Marugan, J., Ferrer, M., Bonifacino, J.S., and Depamphilis, M.L. (2019). A family of PIKFYVE inhibitors with therapeutic potential against autophagy-dependent cancer cells disrupt multiple events in lysosome homeostasis. Autophagy 15, 1694-1718. 10.1080/15548627.2019.1586257. 
She, J., Guo, J., Chen, Q., Zeng, W., Jiang, Y., and Bai, X.-C. (2018). Structural insights into the voltage and phospholipid activation of the mammalian TPC1 channel. Nature 556, 130-134. 10.1038/nature26139.

Stauber, T., and Jentsch, T.J. (2010). Sorting motifs of the endosomal/lysosomal CLC chloride transporters. J Biol Chem 285, 34537-34548. 10.1074/jbc.M110.162545.

Steinberg, B.E., Huynh, K.K., Brodovitch, A., Jabs, S., Stauber, T., Jentsch, T.J., and Grinstein, S. (2010). A cation counterflux supports lysosomal acidification. J Cell Biol 189, 1171-1186. 10.1083/jcb.200911083.

Wang, X., Zhang, X., Dong, X.-P., Samie, M., Li, X., Cheng, X., Goschka, A., Shen, D., Zhou, Y., Harlow, J., et al. (2012). TPC Proteins Are Phosphoinositide- Activated Sodium-Selective Ion Channels in Endosomes and Lysosomes. Cell 151, 372-383. 10.1016/j.cell.2012.08.036.

Wartosch, L., Fuhrmann, J.C., Schweizer, M., Stauber, T., and Jentsch, T.J. (2009). Lysosomal degradation of endocytosed proteins depends on the chloride transport protein CIC-7. FASEB J 23, 4056-4068. 10.1096/fj.09-130880.

Weinert, S., Jabs, S., Hohensee, S., Chan, W.L., Kornak, U., and Jentsch, T.J. (2014). Transport activity and presence of CIC-7/Ostm1 complex account for different cellular functions. EMBO reports 15, 784-791. 10.15252/embr.201438553.

Weinert, S., Jabs, S., Supanchart, C., Schweizer, M., Gimber, N., Richter, M., Rademann, J., Stauber, T., Kornak, U., and Jentsch, T.J. (2010). Lysosomal pathology and osteopetrosis upon loss of H+-driven lysosomal Cl- accumulation. Science 328, 1401-1403. science.1188072 [pii]

Yamamoto, A., Dewald, D.B., Boronenkov, I.V., Anderson, R.A., Emr, S.D., and Koshland, D. (1995). Novel $\mathrm{PI}(4) \mathrm{P}$ 5-kinase homologue, Fab1p, essential for normal vacuole function and morphology in yeast. Molecular Biology of the Cell 6, 525-539. 10.1091/mbc.6.5.525.

Zolov, S.N., Bridges, D., Zhang, Y., Lee, W.-W., Riehle, E., Verma, R., Lenk, G.M., Converso-Baran, K., Weide, T., Albin, R.L., et al. (2012). In vivo, Pikfyve generates PI(3,5)P2, which serves as both a signaling lipid and the major precursor for PI5P. Proceedings of the National Academy of Sciences 109, 1747217477. 10.1073/pnas.1203106109. 